\title{
Gyrofluid Turbulence Models with Kinetic Effects
}

\author{
W. Dorland and G. W. Hammett \\ P. O. Box 451, Princeton, NJ, 08543 \\ Princeton University Plasma Physics Laboratory
}

\begin{abstract}
Nonlinear gyrofluid equations are derived by taking moments of the nonlinear, electrostatic gyrokinetic equation. The principal model presented includes evolution equations tor the guiding center $n, u_{\|}, T_{\|}$, and $T_{\perp}$ along with an equation expressing the quasineutrality constraint. Additional evolution equations for higher moments are derived which may be used if greater accuracy is desired. The moment hierarchy is closed with a Landau-damping model [G. W. Hammett and F. W. Perkins, Phys. Rev. Lett., 64 (1990) 3019] which is equivalent to a multi-pole approximation to the plasma dispersion function, extended to include finite Larmor radius effects. In particular, new dissipative, nonlinear terms are found which model the perpendicular phase-mixing of the distribution function along contours of constant electrostatic potential. These "FLR phase-mixing" terms introduce a hyperviscosity-like damping $\propto k_{\perp}^{2}\left|\Phi_{\vec{k}} \vec{k} \times \vec{k}\right|$ which should provide a physicsbased damping mechanism at high $k_{\perp} \rho$ which is potentially as important as the usual polarization drift nonlinearity. The moments are taken in guiding center space to pick up the correct nonlinear FLR terms and the gyroaveraging of the shear. The equations are solved with a nonlinear, three dimensional initial value code. Linear results are presented, showing excellent agreement with linear gyrokinetic theory.

PACS Numbers: $52.35 . \mathrm{Qz}, 52.25 . \mathrm{Gj}, 52.25 . \mathrm{Fi}, 52.35 . \mathrm{Ra}$
\end{abstract}




\section{Introduction}

\section{A. Overview}

Nonlinear problems in plasma physics have been investigated numerically with a tremendous variety of techniques. These techniques may be very broadly classified into particle simulations, in which many particle trajectories are evolved in time according to simple physical laws, and fluid simulations, in which a few moments of the distribution functin are evolved according to somewhat more complicated relations. The conventional wisdom was that problems which intrinsically involve wave-particle interactions and/or finite Larmor radius (FLR) effects, often referred to as kinetic effects, could not be adequately addressed with fluid techniques, since for these processes the details of the distribution function seem to matter a great deal in the analysis. However, as has been recently shown, ${ }^{1-7}$ many kinetic effects can be included in purely fluid theories when derived with such details in mind. In particular, we recommend Ref. 2 as an introduction to our approach to fluid models of kinetic effects. Furthermore, many authors ${ }^{8-11}$ often utilize fluid theories in nonlinear analyses, because of the relative simplicity and occasional insights afforded by the reduced dynamics described by fluid equations. Finally, numerical solutions of fluid equations may be found using the relatively vast and well-understood arsenal of simulation techniques developed in the computational fluid dynamics and plasma physics communities.

Our long range goal is to derive "gyrofluid" equations (so called because they are derived by taking moments of the gyrokinetic equations and include models of kinetic effects which are missing from traditional fluid equations) with sufficiently accurate models of kinetic effects to simulate the general class of plasma microturbulence which is believed responsible for anomalous transport in tokamaks. In this class of "microturbulence" we include the broad range of instabilities which are covered by the gyrokinetic ordering. ${ }^{12}$ This includes not only the usual trapped-electrondriven drift waves and variants, but also the ion-temperature-gradient (ITG) instability or longer wavelength modes with magnetohydrodynamic (MHD)-type effects, such as the drift-resisitiveballooning mode.

The specific goal of the present paper, however, is to develop effective fluid models of important FLR-induced effects. It is thus sufficient to look at electrostatic perturbations in a sheared slab geometry and assume the electron dynamics are adiabatic. Linearly, these equations contain only slab ITG instabilities ${ }^{13,14}$ and stable electron drift waves, but we intend eventually to extend these equations to the broader range of turbulence spanned by the gyrokinetic ordering. The 
extension to include the toroidal drift resonance necessary for the toroidal version of the ITG instability has been carried out, ${ }^{15,16}$ and we have plans to extend these equations to include the effects of collisions, trapped particles, and fully electromagnetic perturbations. We derive fully nonlinear equations here, though we present in this paper only linear numerical benchmarks. Many issues related to nonlinear effects are addressed here for completeness, such as nonlinear closure assumptions. We will report our nonlinear results in a future publication.

Many authors have considered the problem of including FLR effects in fluid equations for ITG studies. Hamaguchi and Horton ${ }^{17,18}$ developed a "minimum" FLR model and investigated its behavior analytically and numerically using a three-dimensional sheared-slab code which we have used as a platform for our own numerical studies. Lee and Tang $^{19}$ presented fluid equations with a more complete set of FLR effects for analytical investigations of nonlinear conservation properties and mode coupling, but like Hamaguchi and Horton's equations, they are valid only in the $k_{\perp} \rho \ll 1$ limit, and in the absence of magnetic shear. Brizard ${ }^{20}$ has gone quite a bit further, deriving a set of gyrofluid equations in general geometry and including finite- $\beta$ effects, though he assumes that the total distribution function may be considered to be bi-Maxwellian, leading him to overestimate the FLR averaging effects (see Sec. III.C), and to leave higher moments such as the heat fluxes unspecified. The higher moments, however, are in general non-neglible, and must be carefully handled: by neglecting these heat fluxes, one neglects wave-particle effects, such as phase-mixing.

Similon ${ }^{21}$ developed better FLR models which, unlike Refs. 17-19, are linearly exact to all orders in $k_{\perp} \rho$ for a shearless slab geometry. However, his formulation neglected the effects of temperature gradients (because he was concentrating on the usual drift wave) and contained inaccuracies of order $\left(k_{\perp} \rho\right)^{2}$ in the nonlinear and shear terms (see Sec. III.C.1 below). The FLR model employed in Waltz, Dominguez and Hammett ${ }^{15}$ has some similarities to Similon's work (extended to include temperature gradients and the toroidal curvature drive), though it also contains inaccuracies of order $\left(k_{\perp} \rho\right)^{2}$ from $\mathbf{E} \times \mathbf{B}$ nonlinearities and shear. The FLR models developed below regain second order accuracy for small $k_{\perp} \rho$, even when nonlinearities and magnetic shear effects are included. They are also all well behaved at large $k_{\perp} \rho$, much as the Padé approximation $\exp \left(-x^{2}\right) \approx 1 /\left(1+x^{2}\right)$ is both second order accurace for small $\mathrm{x}$ and well behaved for large $x$ [unlike the Taylor Series approximation $\left.\exp \left(-x^{2}\right) \approx 1-x^{2}\right]$. The improved accuracy for nonlinearities and shear is gain $d$ at the expense of losing rigorous higher order accuracy for the shearless linear limit, unless an equal number of parallel velocity moments and perpendicular velocity moments 
are kept (such as in " $3+3$ " model or the " $4+4$ " model shown in the Appendix). Our model is related to Similon's, but we have found that the nonlinear terms are significantly simplified if one takes guiding center fluid moments (in guiding center coordinates), rather than attempting to transform the gyrokinetic equation back to particle coordinates first (also noted independently by Brizard $\left.{ }^{20}\right)$.

A unique feature of our FLR model is its inclusion of a nonlinear FLR-induced phase-mixing effect which arises from the FLR-corrected nonlinear $J_{0}\left(k_{\perp} \rho\right) \mathbf{E} \times \mathbf{B}$ drift. Lehnert ${ }^{22}$ pointed out that this phase-mixing is present in the original kinetic equation in "higher-order orbit theory" than he has so far considered, but did not describe a way to include the effect in fluid equations. High energy particles gyroaverage gradients in the potential more strongly than low energy particles and hence drift more slowly. This spread in drift velocities leads to phase-mixing, a collisionless damping process. Nonlinear gyrokinetic particle sirnulations automatically include this effect, but its significance has not been widely appreciated (excepting Lehnert's work) and its effect has been ignored in previous fluid simulations. This "FLR phase-mixing" leads to a hyperviscositylike damping approximately proportional to $k_{\perp}^{2}\left|\Phi_{\vec{k}} \vec{k} \times \overrightarrow{k^{\prime}}\right|$. Because it is proportional to $\Phi$, it only appears nonlinearly. It provides a physics-based collisionless damping mechanism at high $k_{\perp} \rho$ which is potentially just as important as the usual polarization drift nonlinearity, and may provide an important sink for saturation in a nonlinear, turbulent system.

Ultimately, we foresee a gyrofluid plasma model which would merge the FLR and kinetic models presented here, the toroidal resonance model of Waltz et al. ${ }^{15}$ and Beer, ${ }^{16}$ the fully electromagnetic, general geometry results of Brizard ${ }^{20}$ and the collisional effects considered by Chang and Callen. ${ }^{4,5}$ We hope to understand better the nonlinear dynamics of fusion plasmas by carrying out numerical simulations within this extended fluid paradigm. We view our gyrofluid simulations to be complementary to gyrokinetic particle simulations, as each method will likely prove to have different strengths and weaknesses. Because the gyrofluid equations are derived from the gyrokinetic equation, gyrokinetic particle simulations are more fundamental and potentially more accurate. On the other hand, the reduced dimensionality of the gyrofluid equations (from five to three) should make numerical solutions less recalcitrant. Though it is too early to make quantitative comparisons of efficiency, it has been useful to cross-check and benchmark our linear results. Eventually, we will compare carefully our nonlinear predictions, perhaps providing new physical insight into a difficult problem.

As it stands. our fluid models of kinetic effects may not be adequate for some types of problems, 
especially those in which strongly non-Maxwellian features characterize the distribution function. For such pioblems, it would probably be necessary to keep a large number of fluid moments to describe the detailed shape of the distribution function, in which case the fluid approach may lose its efficiency advantages. For example, we have recently found that the standard nonlinear plasma echo phenomenon can be reproduced with the fluid equations using our phase-mixing closure if the parallel nonlinearities are retained in a fairly large set of equations (we have found that ten parallel velocity moments are needed even for an echo which occurs fairly quickly). ${ }^{23}$ NonMaxwellian features could also result in a given problem if particle trapping in a single electrostatic wave were important, or if (as in the usual quasilinear theory) velocity-space plateaus were created. However, we do not expect such effects to be important in ITG turbulence or for most other types of tokamak microturbulence. The relevant velocity space nonlinearity $\left(\propto E_{\|} \frac{\partial F_{1}}{\partial v_{\|}}\right.$in Eq. (1)) is smaller by a factor of $\varepsilon=\rho / L$ (the gyrokinetic expansion parameter) than the other nonlinear term in Eq. (1). That is,

$$
\frac{\frac{\partial}{\partial v_{\|}}\left(\frac{e}{m} F_{1} \hat{\mathbf{b}} \cdot \nabla J_{0} \Phi\right)}{\nabla \cdot F_{1} J_{0} \mathbf{v}_{E}} \sim \frac{k_{\|} \rho}{k_{\perp} \rho} \sim \varepsilon .
$$

The ordering assumes that the velocity-space gradients of the distribution function $\sim 1 / v_{t}$, and consequently that terms of this size are small. In a strongly turbulent plasma with many interacting modes, each with different resonant particle velocities, it is unlikely that stronger velocity-space gradients can persist. We expect our general approach to be broadly applicable to many outstanding problems in plasma physics; we refer the interested reader to work in progress cited in Ref. 2.

\section{B. Motivation and Outline}

One of the early proposed reasons for the improved confinement of the Supershot regime was the reduction of $L_{n} / L_{T}$, or $\eta_{i}$, with a commensurate reduction of ITG turbulence. However, analysis of the Tokamak Fusion Test Reactor (TFTR) ${ }^{24}$ density perturbation experiments and other TFTR transport studies disproved the reigning concept of marginal $\eta_{i}$ stability and showed that existing transport estimates based on fluid models of slab ITG turbulence were too high by a couple of orders of magnitude. ${ }^{25,26} \mathrm{At}$ about the same time, Kotschenreuther pointed out that existing gyrokinetic ${ }^{27}$ and fluid ${ }^{17}$ estimates of ITG heat transport differed by at least an order of magnitude. ${ }^{27}$ Our gyrofluid model can resolve this disagreement, as we can recover Kotschenreuther's gyrokinetic results by retaining kinetic effects and Hamaguchi and Horton's fluid results 
by neglecting them. The kinetic effects we address are phase-mixing (parallel and perpendicular) and FLR averaging. Although the slab ITG mode is now understood probably to be too weak to explain measured $\chi_{i}$ 's, it appears that the addition of toroidal drive to the ITG mode may raise it back to a level consistent with the core measurements; the theoretical $\gamma_{i}$ is probably still too weak in the outer half of the plasma where other instabilities presumably dominate. ${ }^{26}$ Ultimately, one would prefer to simulate the nonlinear ITG dynamics in a more realistic geometry. Waltz ${ }^{15}$ and Beer ${ }^{16}$ employ models similar to ours in a toroidal setting.

In Section II we review the gyrokinetic Vlasov-Poisson system, the starting point of our derivation. The parallel phase-mixing model we begin with is described in Refs. 1 and 2. This physicallymotivated phase-mixing model includes wave-particle effects such as Landau damping and its inverse, processes which are very important in drift wave physics. In Section III.B we generalize the closure to describe the effects of anisotropic temperature fluctuations, so as to include FLR effects (linear and nonlinear) more easily. In Section III.C, we develop two models of FLR averaging more useful than the usual Taylor series expansions of the gyroaveraging operators for numerical investigations. These models remain robust approximations for $k_{\perp} \rho \geq 1$, where the Taylor series expressions fail. The more accurate " $\Gamma_{0}^{1 / 2}$ " model (Section III.C.2) may be easily added to existing spectral codes, while the "Padé" model (Section III.C.4) could improve the accuracy of finite-difference codes. Both models take into account the gyroaveraging of the shear pointed out by $\mathrm{Bakshi}^{28}$ and Linsker ${ }^{29}$ and recover the adiabatic ion response in the $k_{\perp} \rho \gg 1$ limit. $\mathrm{A}$ new, nonlinear FLR phase-mixing model is described in Section III.D. Lastly, linear results are presented in Sections IV and $\mathrm{V}$, in which we discuss the problem of deciding how many moments it is necessary to retain for the present problem, in the local limit (Section IV) and in the presence of magnetic shear (Section $V$ ). We have utilized the gyrokinetic integral eigenmode code of Linsker ${ }^{29}$ extensively to benchmark the linear performance of our kinetic models.

\section{The Electrostatic Gyrokinetic-Poisson System}

The starting point for our derivation is the nonlinear electrostatic gyrokinetic equation. ${ }^{12,30,31}$ We will find a set of fluid equations by taking velocity-space moments of this equation directly Conceptually, we could begin with the Vlasov equation, generate a set of moments including the stress tensor, heat flux tensor, and so on, and then take the low-frequency $(\omega \ll \Omega)$ limit in the fluid

equations. However, since we are interested in turbulence well-characterized by the gyrokinetic 
ordering, it is natural to take advantage of the strong magnetic field to simplify the kinetic equation first, and then to take the moments. This course has the sizable advantage of retaining the finite Larmor radius (FLR) effects $\left(k_{\perp} \rho \sim 1\right)$ relatively easily. "Gyroviscous cancellations" are recovered automatically with comparatively little effort, even for $k_{\perp} \rho \sim 1$. Also, parallel and perpendicular dynamics (linear and nonlinear) naturally separate, as is appropriate for a collisionless system. The resulting equations are similar to the CGL double-adiabatic equations.

In this paper, we shall consider turbulence in the electrostatic limit, and ignore the effects of non-adiabatic electrons. If electromagnetic perturbations are retained in the derivation below, the reduced MHD equations can be recovered ${ }^{32,20}$ by taking $k_{\perp} \rho \sim \varepsilon$ and ignoring the kinetic phase-mixing model. It is straightforward to add non-adiabatic electrons, velocity gradients or toroidal effects to the present model, extending the class of instabilities which can be modeled beyond the sheared-slab ITG mode.

The usual gyrokinetic ordering is $\omega / \Omega \sim \rho / L \sim k_{\|} \rho \sim F_{1} / F_{0} \sim e \Phi / T_{e} \sim \varepsilon \ll 1$ and $k_{\perp} \rho \sim 1$, where $\omega$ is a typical frequency of the fluctuation spectrum, $\Omega$ is the ion cyclotron frequency, $\rho$ is the ion Larmor radius, $L$ is a typical scale length of the system, $k_{\| 1}$ and $k_{\perp}$ are typical parallel and perpendicular wavenumbers of the fluctuation spectrum, and $\Phi$ is the electrostatic potential.

The nonlinear, electrostatic gyrokinetic equation ${ }^{12,30,31}$ governing the dynamics of the ion distribution function may be written in conservative form as:

$$
\frac{\partial F}{\partial t}+\nabla \cdot\left(F\left(v_{\|} \hat{\mathbf{b}}+J_{0} \mathbf{v}_{E}\right)\right)-\frac{\partial}{\partial v_{\|}}\left(\frac{e}{m} F \hat{\mathbf{b}} \cdot \nabla J_{0} \Phi\right)=0,
$$

where $F\left(\mathbf{R}, v_{\|}, v_{\perp}, t\right)$ is the gyrophase-independent part of the distribution function; that is, it is the density of guiding centers at position $\mathbf{R}$ with parallel velocity $v_{\|}$and perpendicular velocity $v_{\perp}$. $F$ includes both the equilibrium $F_{0}$ and fluctuating $F_{1}$ components, but excludes the gyrophasedependent part of the distribution, given by

$$
\tilde{f}\left(\mathbf{R}, v_{\|}, v_{\perp}, \alpha, t\right)=-\left(\frac{e \Phi(\mathbf{X}, t)}{T_{i}}-\left\langle\frac{e \Phi(\mathbf{X}, t)}{T_{i}}\right\rangle_{\alpha}\right) F_{0},
$$

where the particle position $\mathbf{X}=\mathbf{R}+\rho$ is related to the guiding center position $\mathbf{R}$ and the gyroradius vector $\rho$ (which depends on the gyrophase $\alpha$ ). A veraging over the gyrophase angle $\alpha$ (while holding $\mathbf{R}$ fixed) is denoted by $\langle\cdots\rangle_{\alpha}$. The total distribution function $f\left(\mathbf{R}, v_{\|}, v_{\perp}, \alpha, t\right)=F+\tilde{f}$. where $\dot{f}$ represents an adiabatic response around the gyro-orbit and is related to the polarization drift.

The $\mathbf{E} \times \mathbf{B}$ velocity is given by $\mathbf{v}_{E}=c / B \hat{\mathbf{b}} \times \nabla \Phi, J_{0}$ is a linear operator which carres out 
the gyro-averaging operation. It is simply a Bessel function in Fourier space:

$$
J_{0}\left(\frac{k_{\perp} v_{\perp}}{\Omega}\right)=\frac{1}{2 \pi} \int_{0}^{2 \pi} d \alpha e^{i \frac{k_{\perp} v_{\perp}}{\Omega} \cos \alpha}=\sum_{n=0}^{\infty} \frac{1}{(n !)^{2}}\left(i \frac{k_{\perp} v_{\perp}}{2 \Omega}\right)^{2 n}=\sum_{n=0}^{\infty} \frac{1}{(n !)^{2}}\left(\frac{v_{\perp}}{2 \Omega}\right)^{2 n} \nabla_{\perp}^{2 n} .
$$

In real space, the Bessel function is an operator which may not commute with other operators which appear in the analysis. It is therefore important to keep track of what has been gyroaveraged (and therefore what is being operated upon by a given $J_{0}$ ) at each point in the derivation. $J_{0}$ operates only on $\Phi$ in Eq. (1).

Note that this ordering retains the physics of strong turbulence even though the fluctuating quantities are ordered small, since their derivatives may be $O(1)$. That is, although $F_{1} / F_{0} \sim \varepsilon$, $\left(\nabla_{\perp} F_{1}\right) /\left(\nabla_{\perp} F_{0}\right) \sim 1$, thus retaining the dominant $\mathbf{E} \times \mathbf{B}$ nonlinearity.

This equation is closed by the quasineutrality constraint $n_{i}(\mathbf{x})=n_{e}(\mathbf{x})$, which when written in terms of the guiding center quantities becomes

$$
\bar{n}_{i}-n_{i 0}\left(1-\Gamma_{0}\right) \frac{e \Phi}{T_{i}}=n_{e}
$$

in which $\Gamma_{n}(b)=I_{n}(b) e^{-b}$ and $I_{n}(b)=i^{-n} J_{n}(i b)$ is the modified Bessel function. $\bar{n}_{i}(\mathbf{x})$ is the contribution to the local particle density from the gyrophase-independent part of the guiding-center distribution function $F$,

$$
\bar{n}_{\boldsymbol{i}}(\mathbf{x})=\int d^{3} v J_{0} F=\int d^{3} v\left(F_{0}+J_{0} F_{1}\right) .
$$

In this expression, $J_{0}$ operates on the distribution function (though $F_{0}$ is slowly varying so $J_{0} F_{0} \approx$ $F_{0}$ ). The second term on the left-hand side of Eq. (3) (usually called the "polarization density") is the contribution to the local particle density from $\tilde{f}$, the gyrophase-dependent part of the distribution function.

We can use the same fluid equations derived below for the ions to describe the electron dynamics, usually also assuming $k_{\perp} \rho_{e} \ll 1$. However, in this paper we shall consider only the adiabatic part of the electron response, and thus Eq. (3) becomes

$$
\vec{n}_{i}-n_{i 0}\left(1-\Gamma_{0}\right) \frac{e \Phi}{T_{i}}=n_{e 0}\left[1+\frac{|e|}{T_{e}}(\Phi-\ll \Phi \gg)\right] .
$$

The $\ll \Phi \gg$ ) term on the right-hand side represents the flux surface average of the electrostatic potential,

$$
\ll \Phi \gg=\frac{1}{L_{y} L_{z}} \int_{0}^{L_{y}} d y \int_{0}^{L_{x}} d z \Phi
$$

and must be included to prevent non-physical electron transport across the flux surface. 


\section{Derivation of the Gyrofluid Equations}

\section{A. General Gyrofluid Equations}

We choose to take the moment averages in guiding center coordinates. Thus $n$ is the density of guiding cencers, $m n u_{\|}$is the momentum of guiding centers, etc. Specifically,

$$
\begin{array}{cc}
n \equiv \int F d^{3} v & n u_{\|} \equiv \int F v_{\|} d^{3} v \\
p_{\perp} \equiv(m / 2) \int F v_{\perp}^{2} d^{3} v & p_{\|} \equiv m \int F\left(v_{\|}-u_{\|}\right)^{2} d^{3} v \\
q_{\perp} \equiv(m / 2) \int F v_{\perp}^{2}\left(v_{\|}-u_{\|}\right) d^{3} v & q_{\|} \equiv m \int F\left(v_{\|}-u_{\|}\right)^{3} d^{3} v \\
r_{\perp} \equiv(m / 2) \int F v_{\perp}^{2}\left(v_{\|}-u_{\|}\right)^{2} d^{3} v & r_{\|} \equiv m \int F\left(v_{\|}-u_{\|}\right)^{4} d^{3} v \\
s_{\perp} \equiv(m / 2) \int F v_{\perp}^{2}\left(v_{\|}-u_{\|}\right)^{3} d^{3} v .
\end{array}
$$

Using these definitions and the relations $p_{\|, \perp}=n T_{\|, \perp}$, we may derive the following general fluid equations by taking various integrals of the form $\int d^{3} v v_{\perp}^{2 j} v_{\|}^{\ell} \cdots$ over Eq. (1):

$$
\begin{aligned}
& \frac{\partial n}{\partial t}+\nabla \cdot\left(n u_{\|} \hat{\mathbf{b}}+n\left\langle J_{0}\right\rangle \mathbf{v}_{E}\right)=0 \\
& \frac{\partial n u_{\|}}{\partial t}+\nabla \cdot\left(\left(\frac{p_{\|}}{m}+n u_{\|}^{2}\right) \hat{\mathbf{b}}+n\left\langle v_{\|} J_{0}\right\rangle \mathbf{v}_{E}\right)+\frac{\dot{e}}{m} \hat{\mathbf{b}} \cdot n\left\langle J_{0}\right\rangle \nabla \Phi=0 \\
& \frac{\partial\left(p_{\|}+m n u_{\|}^{2}\right)}{\partial t}+\nabla \cdot\left(\left(q_{\|}+3 p_{\|} u_{\|}+m n u_{\|}^{3}\right) \hat{\mathbf{b}}+n\left\langle m v_{\|}^{2} J_{0}\right\rangle \mathbf{v}_{E}\right) \\
& +2 e \hat{\mathbf{b}} \cdot n\left\langle v_{\|} J_{0}\right\rangle \nabla \Phi=0 \\
& \frac{\partial\left(q_{\|}+3 u_{\|} p_{\|}+m n u_{\|}^{3}\right)}{\partial t}+\nabla \cdot\left(\left(r_{\|}+4 u_{\|} q_{\|}+6 u_{\|}^{2} p_{\|}+m n u_{\|}^{4}\right) \hat{\mathbf{b}}+n\left\langle m v_{\|}^{3} J_{0}\right\rangle \mathbf{v}_{E}\right) \\
& +3 e \hat{\mathbf{b}} \cdot n\left\langle v_{\|}^{2} J_{0}\right\rangle \nabla \Phi=0 \\
& \frac{\partial p_{\perp}}{\partial t}+\nabla \cdot\left(\left(q_{\perp}+n u_{\|} T_{\perp}\right) \hat{\mathbf{b}}+n\left\langle\frac{m v_{\perp}^{2}}{2} J_{0}\right\rangle \mathbf{v}_{E}\right)=0 \\
& \frac{\partial\left(q_{\perp}+n u_{\|} T_{\perp}\right)}{\partial t}+\nabla \cdot\left(\left(r_{\perp}+2 u_{\|} q_{\perp}+n u_{\|}^{2} T_{\perp}\right) \hat{\mathbf{b}}+n\left\langle v_{\|}\left(\frac{m v_{\perp}^{2}}{2}\right) J_{0}\right\rangle \mathbf{v}_{E}\right) \\
& +\frac{e}{m} n\left\langle\left(\frac{m v_{\perp}^{2}}{2}\right) J_{0}\right\rangle \hat{\mathbf{b}} \cdot \nabla \Phi=0 \text {. }
\end{aligned}
$$


where $\langle\cdots\rangle \equiv \int d^{3} v F(\cdots) / \int d^{3} v F$. These equations are the exact, nonlinear evolution equations for the first few moments of the electrostatic gyrokinetic equation in the collisionless limit. They express the conservation of density, parallel momentum, parallel and perpendicular energy, and so on. However, their utility is limited unless closure approximations are specified. In this case, we must make two distinct closure assumptions.

First, we must close the usual fluid hierarchy which results from the linear parallel convection term $\left(\nabla \cdot\left(F v_{\|} \hat{\mathbf{b}}\right)\right.$ in the gyrokinetic equation). Because of this term, the time evolution of each fluid moment is driven in part by the gradient of the next-higher parallel velocity moment. It has been shown ${ }^{1,2}$ that collisionless phase-mixing (and thus Landau damping) may be modeled $b_{y}$ approximating this single term in the highest moment equations retained. Note that in the present case, we will find expressions for $r_{\|}$and $r_{\perp}$ which appear in Eqs. (10) and (12), respectively. No dissipation is introduced into the evolution equations for the lower moments by this closure, which therefore remain exact nonlinear expressions. Later in this particular derivation, however, we do neglect the higher-order parallel nonlinearities, as noted in the Introduction.

Second, we must close each of the FLR terms $\left\langle\cdots J_{0}\right\rangle$ which arise from the gyroaveraging process. The gyroaveraging operator $J_{0}$ depends in general upon all even powers of $v_{\perp}$, as is evident from Eq. (2). Thus, $\left\langle J_{0}\right\rangle$ is in general a function of all $v_{\perp}^{2 j}$ moments of the distribution function. We will approximate these higher moments in terms of moments which we evolve in time. Furthermore, the nonlinear part of the $J_{0} \mathbf{v}_{E} \cdot \nabla$ term in Eq. (1) is responsible for perpendicular FLR phase-mixing analogous to the parallel phase-mixing which arises from the $v_{\|} \nabla_{\|}$term. The closure approximations for the various $\left\langle\cdots J_{0}\right\rangle$ terms may be chosen to model this collisionless damping process. To our knowledge, all previous fluid closure approximations for the FLR terms have missed this effect.

We will discuss the closure approximations for various parallel and FLR terms in the next several sections. The final gyrofluid equations are given in Sec. III.E.

\section{B. Parallel Phase-Mixing Closure}

The parallel phase-mixing model outlined in Ref. 1 is immediately appropriate for the parallel fluid moments (such as $r_{\| 1}$ ), but does not directly address the effects of anisotropic temperature perturbations, which for $k_{\perp} \rho \sim 1$, are as important as the parallel temperature fluctuations and should be treated on an equal footing. $T_{\perp}$ appears linearly in Poisson's equation and nonlinearly 
in the density equatir $\mathrm{n}$ at the same order as each of the other termis retained. Rather than using only one equation to express the evolution of the total temperature $T=\left(2 T_{\perp}+T_{11}\right) / 3$ and trying to correct for the difference between $T_{\perp}$ and $T_{\|}$with an approximate stress tensor, we choose to evolve two separate equations for the parallel and perpendicular temperatures. This route allows one to find the nonlinear FLR corrections $\left(\propto T_{\perp}\right)$ easily, and bypasses the tedious algebra associated with the gyroviscous corrections. Because the perpendicular temperature evolution equation includes the same kind of parallel resonance term as is found in each of the other equations, one expects to find a similarity in the parallel phase-mixing closures. In fact, the underlying kinetic equation guarantees the connection between the parallel moments $\left(n, u_{\|}, T_{\|}\right.$and $\left.q_{\|}\right)$and the perpendicular moments $\left(T_{\perp}, q_{\perp}, r_{\perp}\right.$ and $\left.s_{\perp}\right)$ which we exploit in Section III.C.

The closure prescription in Ref. 1 is to approximate the highest moments which occur in the fluid hierarchy with the Maxwellian part of each plus a small correction due to the perturbed part of the distribution function, chosen to reproduce the linear response function $R(\zeta)=-\tilde{n} /\left(n_{0} e \Phi / T_{0}\right)$ in the low- and high-frequency limits. However, examination of Eqs. (7-12) reveals that linearly when $k_{\perp} \rho \ll 1$, the perpendicular moment fluctuations are decoupled from the density perturbations. Rather than trying to solve for the closure of the FLR terms and the phase-mixing terms all at once, we will take advantage of this decoupling and consider the problem in a slightly different light.

\section{Linear Propagator}

Consider a simple one-dimensional, 1. smogeneous system in the absence of particle interactions. Linearly, the perturbed distribution function evolves according to

$$
\frac{\partial F_{1}}{\partial t}+v \frac{\partial F_{1}}{\partial z}=\delta(t) g(z, v)
$$

where $g$ is a perturbation assumed to occur at time $t=0$. It is convenient to define the kinetic linear propagator $\mathcal{L}$, whose inverse can be represented in Fourier space as

$$
\mathcal{L}_{k}^{-1} \equiv-i \omega+i k v
$$

so that $F_{1 k}=\mathcal{L}_{k} g_{k}$ and we must use the Landau prescription for the singularity on the real axis. For the moment, we consider the case in which $g_{k}(v)$ is Maxwellian with a perturbation only in the density. Note that previously ${ }^{1}$ we considered the case in which $g \propto v F_{M}(v) \propto E_{\|} \partial F_{. M} / \partial v_{\|}$, as is appropriate for an electric field term in the usual Landau damping problem. Here we show that 
one finds the same closure coefficients for the case in which the initial perturbation $g \propto F_{M}(v)$. This is not a coincidence; rather, it is related to the fact that the solution to Eq. (13), $F_{1 k}=$ $F_{M}(v) e^{i k(z-v t)}$, contains products of $F_{M}$ and all powers of the velocity $v^{n}$ as time goes on:

$$
F_{. M}(v) e^{-i k v t} \simeq F_{. M}(v)\left(1-i k v t-(k t)^{2} \frac{v^{2}}{2}+\cdots\right)
$$

The density response in the frequency domain is simply

$$
\frac{n_{1 k}}{n_{0}}=\frac{1}{n_{0}} \int d^{3} v F_{1}=\frac{1}{n_{0}} \int d^{3} v F_{M} \mathcal{L}_{k}=-\frac{i \zeta}{\omega} Z(\zeta),
$$

in which $\zeta=\omega / \sqrt{2}\left|k_{\|}\right| v_{t}, v_{t}=\sqrt{T / m}$ and $Z(\zeta)$ is the usual plasma dispersion function. We may recast the closure scheme in Ref. 1 in terms of the lowest moments of the linear propagator. From this viewpoint, the generalization to anisotropic temperature fluctuations will be trivial.

For simplicity, consider a two-moment fluid model, $n$ and $u_{\| 1}$, of the same system. The linearized fluid equations written in nondimensional units (see Eqs. (53) and (54)) are

$$
\begin{array}{r}
\frac{\partial n}{\partial t}+\nabla_{\|} u_{\|}=\delta(t) S(z) \\
\frac{\partial u_{\|}}{\partial t}+\nabla_{\|} p_{\|}=0,
\end{array}
$$

in which $S(z)=\int d v g(z, v)$. Following Ref. 1, we approximate $p_{\|}$in terms of the lower moments $n$ and $u_{\|}$:

$$
p_{\|} \simeq \beta n-\mu \nabla_{\|} u_{\|}
$$

and Fourier transform the resulting equations to ind the appropriate closure relations for $\beta$ and $\mu$. Anticipating the result, the form of the collisionless diffusion coefficient $\mu$ for this simple fluid model is taken to be

$$
\mu \equiv \mu_{1} \frac{\sqrt{2}}{\left|k_{\|}\right|}
$$

in Fourier space, and by an integral operator in real space. ${ }^{1} \beta$ and $\mu_{1}$ are dimensionless parameters of $O(1)$ to be determined.

Using Eq. (17) to find $u_{\|}$and substituting the result into Eq. (16) leads to

$$
\left[-i \omega+\frac{i k_{\|}^{2} \beta}{\omega+i \mu_{1} \sqrt{2}\left|k_{\|}\right|}\right] n=S \text {. }
$$

We may use this to define the linear propagator for density, $n=\mathcal{L}_{n} S$. We may rewrite $\mathcal{L}_{n}$ in the form:

$$
\mathcal{L}_{n}=\frac{i \zeta}{\omega}\left(\frac{\zeta+i \mu_{1}}{\zeta^{2}+i \mu_{1} \zeta-3 / 2}\right)=-\frac{i \zeta}{\omega} Z_{2}(\zeta)
$$


The closure coefficients $\mu_{1}$ and $\beta$ are determined by requiring the density propagator (Eq. (19)) to match the zeroth moment of the kinetic propagator (Eq. (15)) in both the "adiabatic" $(\zeta \ll 1)$ and "fluid" $(\zeta \gg 1)$ limits. The resulting function $Z_{2}(\zeta)$ is a two-pole approximation to the plasma dispersion function $Z(\zeta)$, shown in Fig. 1. We find that $\beta=1$ and $\mu_{1}=\sqrt{\pi} / 2$.

\section{Anisotropic Temperature Fluctuations}

Since this formalism makes no reference to the presence of the electric field, it generalizes trivially to the case of fuctuations of the perpendicular temperature (which are decoupled from the potential fluctuations in the $k_{\perp} \rho \ll 1$ limit). To isolate the closure terms for the perpendicular moments, we consider the case in which $g\left(z, v_{\|}, v_{\perp}\right)$ in Eq. (13) is bi-Maxwellian with a perturbation in $T_{\perp}$ only, and not in $n$. That is,

$$
g\left(z, v_{\|}, v_{\perp}\right)=F_{m}\left(v_{\|}, v_{\perp}\right)\left[1+\left(\frac{m v_{\perp}^{2}}{2 T_{\perp 0}}-1\right) S_{\perp}(z)\right] .
$$

The fluid equations analogous to Eqs. (16) and (17) are:

$$
\begin{aligned}
& \frac{\partial T_{\perp}}{\partial t}+\nabla_{\|} q_{\perp}=\delta(t) S_{\perp}(z) \\
& \frac{\partial q_{\perp}}{\partial t}+\nabla_{\|} r_{\perp}=0 .
\end{aligned}
$$

As before, the appropriate normalizations are given in Eqs. (53) and (54). As before, we approximate the highest moment $r_{\perp}$ in terms of the lower moments $T_{\perp}$ and $q_{\perp}$,

$$
r_{\perp} \simeq \beta T_{\perp}-\mu \nabla_{\|} q_{\perp}
$$

and Fourier transform the resulting equations. Note that we are now interested in the linear propagator for the perpendicular temperature fluctuation,

$$
\mathcal{L}_{T}^{-1} \equiv-i \omega+\frac{i k_{\|}^{2} \beta}{\omega+i \mu_{1} \sqrt{2}\left|k_{\|}\right|}
$$

which we may write as

$$
\mathcal{L}_{T}=-\frac{i \zeta}{\omega} Z_{2}(\zeta)
$$

It is now easily shown that we should choose $\beta$ and $\mu$ exactly as before. Since the kinetic linear propagator $\mathcal{L}$ is independent of $v_{\perp}$, the second perpendicular moment of $\mathcal{L}^{-1}$ is exactly the same as was found in Eq. (15) (in non-dimensional units). This is not surprising, since the underlying kinetic resonance is the same for the hierarchy of parallel moments $\left(n, u_{\|}, \ldots\right)$ as for the hierarchy 
of perpendicular moments $\left(T_{\perp}, q_{\perp}, \ldots\right)$. Eq. (22), with $\beta=1$ and $\mu=\sqrt{\pi / 2} /\left|k_{\|}\right|$, provides the closure for $r_{\perp}$ in Eq. (12).

Keeping the evolution equations for $n$ coupled moments leads in this way to $n$-pole Padé approximants for the plasma dispersion function. For analytic investi" 'ions, it is usually convenient to keep an equal number of parallel and perpendicular momentw, as all of the $Z$-function approximations which appear are then the same.

\section{Moment Reduction Scheme}

In Ref. 2 we presented a 1-moment model, "the simplest possibie fluid model of phase-mixing". That model was Eq. (16) with the closure approximation $u_{\|}=-\left(\nu_{1} /\left|k_{\|}\right|\right) \nabla_{\|} n$, and $\nu_{1}=\sqrt{2 / \pi}$. Note that this same expression for $u_{\|}$can be obtained by taking the $k_{\|} \gg \omega$ limit of Eq. (17) (with the closure for $p_{\|}$given by Eq. (18)). We will use this fact to generalize the 1-moment model to include some additional adiabatic and FLR effects.

In its current form, the 1-moment model makes no reference to the electric field, so it will not recover the adiabatic limit $\left(\omega \ll k_{\| 1}\right)$ where the density should be proportional to $\Phi, n=-\Phi$. The problem is that $\Phi$ appears explicitly only in the $\partial u_{\|} / \partial t$ equation, so a closure approximation in a lower moment equation won't include the effects of $\Phi$ unless the closure is modified in some way. One way to do this is to go back to Eq. (17) and include the electrostatic potential on the right hand side. With Eq. (18), Eq. (17) then becomes

$$
\frac{\partial u_{\|}}{\partial t}+\nabla_{\|} n+\mu_{1} \sqrt{2}\left|k_{\|}\right| u_{\|}=-\nabla_{\|} \Phi
$$

Taking the large $k_{\|}$limit of this equation leads to a new closure approximation for $u_{\|}$which includes the effect of the electric potential, $u_{\|}=-\left(\nu_{1} /\left|k_{\|}\right|\right) \nabla_{\|}(n+\Phi)$. Inserting this closure into Eq. (16) causes the density to relax to the adiabatic limit. This mends the deficiency with a result we might have guessed, since we always expect to recover the adiabatic response in the zero-frequency limit.

This procedure can be generalized to derive an $n-1$ moment closure from an $n$-moment closure. The basic idea is that by taking the high $k_{\|}$limit of the $n^{\text {th }}$ fluid moment equation, then the $\partial / \partial t$ becomes negligible and one obtains a frequency independent closure approximation for the $n^{\text {th }}$ moment which can be used in the $n-1$ moment equation. Physically, this is related to the fact that phase-mixing is most important for $\omega \ll k_{\|} v_{t}$, which is where the closure can be determined. 
The opposite limit, $\omega \gg k_{\|} v_{t}$, is the cold-plasma limit where the highest moment can be ignored and the choice of closure is not too important.

For example, the 2-moment closure for $p_{\|}$in Eq. (18) can be derived by taking the large $k_{\|}$ limit $\left(k_{\|} v_{t} \gg \omega, \omega\right.$ ) of the 3-moment equation for $T_{\|}$, Eq. (65). In turn, the 3-moment closure for $q_{\|}$used in Eq. (65) can be derived by taking the large $k_{\|}$limit of the 4-moment equation for $q_{\|}$, Eq. (59). Likewise, taking the high $k_{\|}$limit of Eq. (61) leads to the closure approximation

$$
q_{\perp}=-\frac{1}{\sqrt{2} D_{\perp}\left|k_{\|}\right|} \nabla_{\|}\left(T_{\perp}+\frac{1}{2} \dot{\nabla}_{\perp}^{2} \Psi\right)
$$

where $\nabla_{\perp}^{2} \Psi \approx \nabla_{\perp}^{2} \Phi$ includes FLR-related adiabatic terms in the $T_{\perp}$ dynamics. This closure for $q_{\perp}$ is used in the " $3+1$ " model of Eqs. (63-66), which uses 3 parallel moments but only 1 perpendicular moment. In the the Appendix, we present an 8 moment gyrofluid model, which we refer to as the " $4+4$ " model, since it contains 4 parallel moments $\left(n, u_{\|}, T_{\|}\right.$, and $\left.q_{\|}\right)$and 4 perpendicular moments $\left(T_{\perp}, q_{\perp}, r_{\perp}\right.$, and $\left.s_{\perp}\right)$. Each of our lower moment models can easily be obtained from this " $4+4$ " set.

One caveat should be mentioned. The $n \rightarrow n-1$ moment reduction procedure outlined in this section must be modified if there are more than 4 parallel or perpendicular moments. For $n>4$, care must be taken about the choice of decomposing the highest moment into reducible and irreducible parts (e.g. Eq. (23)). Only one choice will lead to the proper reduction from $n$ moments to $n-1$ moments.

\section{Parallel Phase-Mixing Closure Summary}

We implement the parallel phase-mixing closure in Eqs. (10) and (12) by approximating the highest two full moments, $r_{\|}$and $r_{\perp}$ in terms of the lower moments. We find it useful first to write each in terms of its reducible components plus an irreducible correction:

$$
r_{\|} \equiv \frac{3 p_{\|}^{2}}{m n}+\delta r_{\|} \quad \text { and } \quad r_{\perp} \equiv \frac{p_{\|} p_{\perp}}{m n}+\delta r_{\perp}
$$

This step simplifies the later algebra without changing the final result. We then linearize, and approximate the irreducible part so as to reproduce the low- and high-frequency limits of the lowest moments of the linear propagator. The closure approximation has a simple form in wave-number space. That is, in dimensional units,

$$
\delta r_{\|} \equiv-D_{\|} \frac{\sqrt{2} v_{t}}{\left|k_{\|}\right|} i k_{\|} q_{\| 1}+\beta_{\|} \frac{p_{\perp 0}}{m} T_{\| 1} \quad \text { and } \quad \delta r_{\perp} \equiv-D_{\perp} \frac{\sqrt{2} v_{t}}{\left|k_{\|}\right|} i k_{\|} q_{\perp}+3_{\perp} \frac{p_{\| 0}}{m} T_{\perp 1} \text {. }
$$


The constants $D_{\|}, D_{\perp}, \beta_{\|}$and $\beta_{\perp}$ are:

$$
\begin{array}{ll}
D_{\|} \equiv \frac{2 \sqrt{\pi}}{3 \pi-8} & D_{\perp} \equiv \frac{\sqrt{\pi}}{2} \\
\beta_{\|} \equiv \frac{32-9 \pi}{3 \pi-8} & \beta_{\perp} \equiv 1,
\end{array}
$$

exactly as found in Refs. 1 and 2. Figs. 1 and 2 show the real and imaginary parts of the local kinetic response function for a Maxwellian $F_{0}, R(\zeta)=1+\zeta Z(\zeta)$ compared with our fluid approximations.
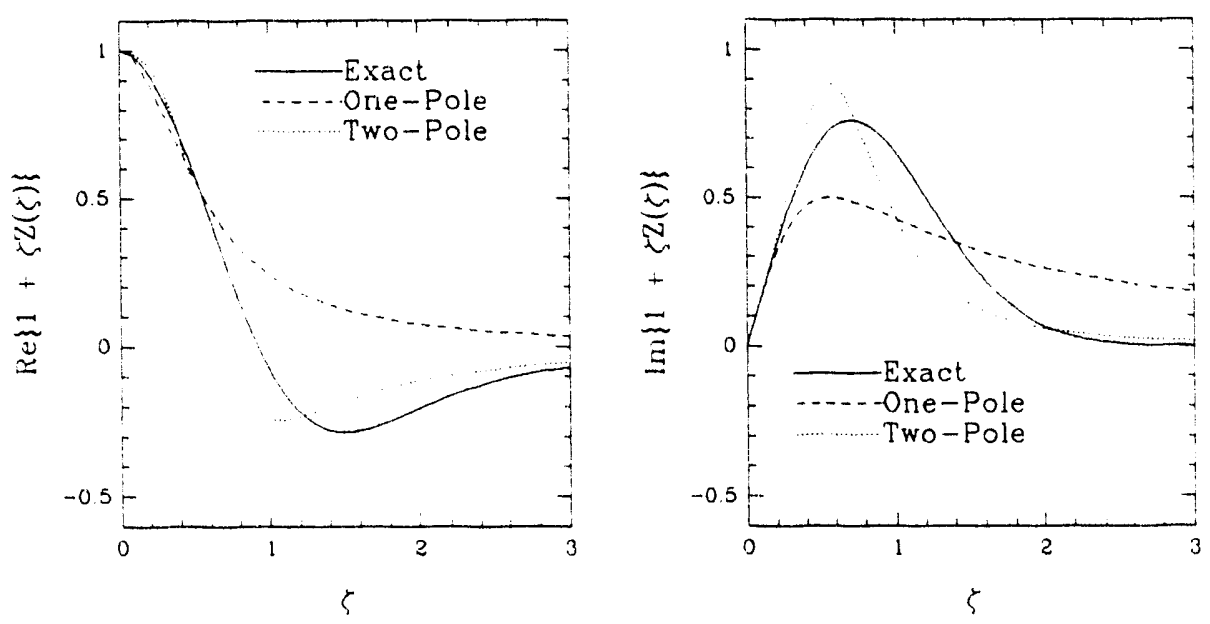

Figure 1: $1+\zeta Z(\zeta)$ for one- and two-pole models of parallel phase-mixing.

In these figures, the argument of the plasma dispersion function is real. The approximations to the collisionless response in all but the one-pole models are reasonable, improving as more moments are kept. Each reproduces the the most important qualitative features of the response. The one-pole model is the roughest for while it is correct at $\zeta=0$ and $\zeta=\infty$ and gives damping for intermediate values of $\zeta$, the real part of the response function has the wrong sign for $\zeta>1$.

Most importantly, our approach eliminates the singularities which are present at resonances in the collisionless limit of most previous fluid equations. These closure relations are exact for some distribution which is close to Maxwellian ${ }^{1}$ (but only approximate in the case that the equilibrium distribution function is Maxwellian). It is therefore not surprising that Landau damping and its inverse, ion Compton scattering and other "kinetic" phenomena are described reasonably well by these few equations. 

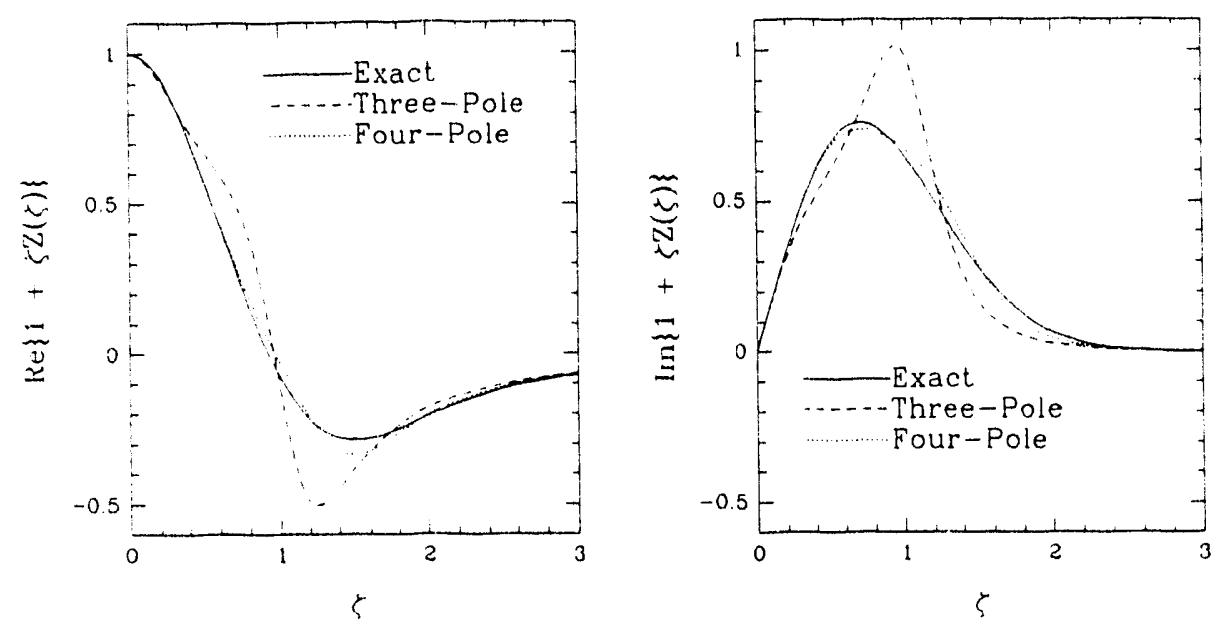

Figure 2: $1+\zeta Z(\zeta)$ for three- and four-pole models of parallel phase-mixing.

\section{Finite Larmor Radius Effects}

We now investigate the conventional (non-dissipative) FLR effects buried in the various integrals involving $J_{0}$ in the fluid equations, Eqs. (7-12). (The collisionless dissipation induced by FLR will be considered in Section III.D.) These integrals are of the form

$$
\int d^{3} v v_{\perp}^{2 j} v_{\| 1}^{\ell} F J_{0}=n\left\langle v_{\perp}^{2 j} v_{\|}^{\ell} J_{0}\right\rangle
$$

Note that an exact, fully nonlinear $\left(\operatorname{arbitrary} F\right.$ ) evaluation of $\left\langle J_{0}\right\rangle$ would require an infinite set of velocity space moments, since $J_{0}$ contains all even powers of $v_{\perp}$; approximations are therefore necessary.

The standard second-order Taylor series expansion of $J_{0}$, yields $\left\langle J_{0}\right\rangle \simeq 1-b / 2$, in which $b=\rho_{i}^{2} k_{\perp}^{2}$ and $\rho_{i}^{2}=\left\langle v_{\perp}^{2}\right\rangle /(2 \Omega)=T_{\perp} /(m \Omega)$. This approximation is equivalent to assuming that each of the perpendicular moments higher than $\left\langle v_{\perp}^{2}\right\rangle$ is identically zero. In addition to the various $J_{0}$ integrals in the fluid equations where $J_{0}$ operates on $\Phi$, we must also evaluate Eq. (4), in which $J_{0}$ operates on $F$ itself. A Taylor series expansion of the $J_{0}$ in Eq. (4) yields

$$
\bar{n}=\int d^{3} v J_{0} F=n+\nabla_{\perp}^{2}\left(n \frac{T_{\perp}}{m 2 \Omega^{2}}\right) .
$$

Expanding $n T_{\perp}$ in the last term into equilibrium and perturbed components in the gyrokinetic ordering (where, for example, $n_{1} \ll n_{0}$ but $\nabla_{\perp} n_{1} \sim \nabla_{\perp} n_{0}$ ) leads to

$$
\tilde{n}_{1}=\left(1-\frac{b_{0}}{2}\right) n_{1}-\frac{n_{0}}{T_{\perp 0}} \frac{b_{0}}{2} T_{\perp 1} \text {, }
$$




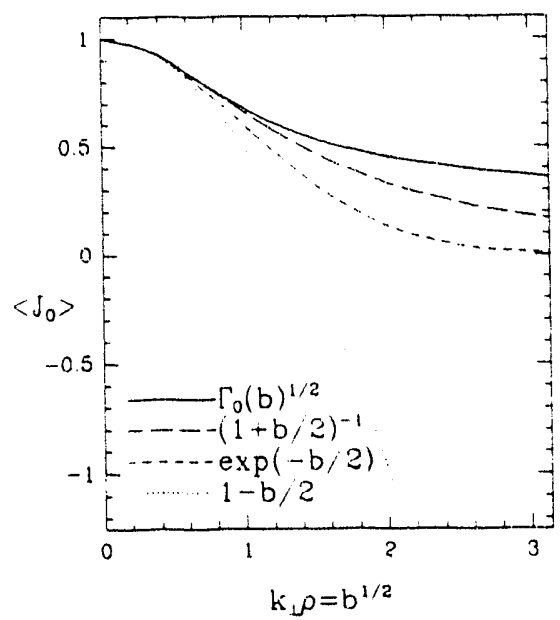

Figure 3: Comparison of FLR approximations to $\left\langle J_{0}\right\rangle$.

where $b_{0}=k_{\perp}^{2} T_{\perp 0} /\left(m \Omega^{2}\right)$. The Taylor series approach provides the simplest second-order accurate model. It is useful for developing analytic understanding in the $k_{\perp} ? \ll 1$ limit, but should be used cautiously because of the large errors introduced for $k_{\perp} \rho>1$. The errors are particularly important in numerical simulations, since the typical grid needed to resolve the dynamics introduces large $k_{\perp} \rho$ values into the system. That is, the grid spacing in the direction of the background gradient must be $\Delta x \sim \rho$, which implies that the maximum $k_{x} \rho$ present is $\sim \pi$. The Taylor series approximation is egregiously wrong for such wavenumbers, since $1-b / 2 \approx-4$, while $J_{0}$ is bounded between -0.4 and 1. In Fig. 3, we compare different expressions for $\left\langle J_{0}\right\rangle$. The error in the Taylor series approximation for the $\left\langle J_{0}\right\rangle$ FLR terms is unarceptably large for $k_{\perp} \rho>\sqrt{2}$. The Taylor series expansion of $\Gamma_{0}(b)=1-b$ (which appears in the quasineutrality constraint, Eq. (3)) goes awry even sooner, as it is negative for $k_{\perp} \rho>1$, while $\Gamma_{0}(b)$ is always positive. This can cause significant errors in the linear growth rates $i$. 1 d eigenmode shapes, as demonstrated in Figs. 9-11, which will be discussed in Secs. IV and V.

Another way to carry out the various $J_{0}$ integrals is to assume that the total guiding center $F$ is always bi-Maxwellian (with perturbations in $n, T_{\perp}, u_{\|}$, and $T_{\|}$). This would imply, for example, that

$$
\left\langle J_{0}\right\rangle=e^{-b / 2}=1+\frac{T_{\perp}}{2 m \Omega^{2}} \nabla_{\perp}^{2}+\frac{T_{\perp}^{2}}{8 m^{2} \Omega^{4}} \nabla_{\perp}^{4}+\cdots
$$

and so on, where $b=b_{0}+b_{1} \propto T_{\perp 0}+T_{\perp 1}$ contains both equilibrium and perturbed parts of the $T_{\perp}$. We remind the reader that the $\nabla_{\perp}^{2}$ operators above operate only on $\Phi$ in Eqs. (7-12). Applying 
the same assumption that $F$ is always bi-Maxwellian to Eq. (4), where $J_{0}$ operates on $F$ itself, leads to

$$
\bar{n}_{1}=\int d^{3} v J_{0} F-n_{0}=e^{-b_{0} / 2} n_{1}+\frac{n_{0}}{T_{\perp 0}} \frac{\rho_{0}^{2}}{2} e^{-b_{0} / 2} \nabla_{\perp}^{2} T_{\perp 1} .
$$

This "Maxwellian total $F$ ", or $\left\langle J_{0}\right\rangle \approx \exp (-b / 2)$, approximation reduces to the second-order Taylor series approximation in the small $k_{\perp} \rho$ limit, but it is better behaved for large $k_{\perp} \rho$. That is, it gives the reasonable result that $\left\langle J_{0}\right\rangle \rightarrow 0$ as $k_{\perp} \rho \rightarrow \infty$. We tried this approach at an early stage in our research (an approach used independently by Brizard ${ }^{20}$ ), but discovered that it did not match the kinetic linear dispersion relation very well for $k_{\perp} \rho \sim 1$ or larger (e.g., see Fig. 9). The reason is that although the equilibrium $F_{0}$ is Maxwellian, the linear perturbation $F_{1}$ is quite non-Maxwellian in $v_{\perp}$. Indeed, the standard linear solution of Eq. (1) in an unsheared slab gives $F_{1} \propto F_{0} J_{0}\left(\alpha+v_{\perp}^{2} \beta\right)$. Inserting this into $\mathrm{Eq}$. (4) yields a linear dispersion relation with terms proportional to $\int d^{3} v\left(F_{0} / n_{0}\right) J_{0}^{2}=\left\langle J_{0}^{2}\right\rangle_{0}=\Gamma_{0}\left(b_{0}\right)$. This is quite different from the case where the total $F$ (including $F_{1}$ ) is assumed Maxwellian, where the $\left\langle J_{0}\right\rangle=\exp (-b / 2)$ in the fluid equations combines with another factor of $\exp (-b / 2)$ in the $\bar{n}_{i}$ calculation to give terms proportional to $\exp (-b)$. Since the correct kinetic result, $\Gamma_{0}(b)$, has a much weaker $b$ dependence at large $b$ (asymptotically proportional to $1 / \sqrt{b}$ ), the "Maxwellian total F" approximation results in too much gyroaveraging at large $b$. This major difference between $\left\langle J_{0}\right\rangle^{2}$ and $\left\langle J_{0}^{2}\right\rangle$ is part of what motivated the $\left\langle J_{0}\right\rangle \approx\left\langle J_{0}^{2}\right\rangle^{1 / 2}=\Gamma_{0}^{1 / 2}$ model considered below. We shall find that it is linearly correct to all orders in $k_{\perp} \rho$ in the absence of magnetic shear and nonlinearly second-order accurate in its presence. But first we consider Similon's approach.

\section{Simiion and the Particle Space Approach.}

Similon ${ }^{21}$ derived a set of fluid equations for the nonlinear electron drift wave problem. Since the quasineutrality constraint depends directly on the particle density $\bar{n}=\int d^{3} v J_{0} F$ (plus the polarization part) and not on the guiding center density $n=\int d^{3} v F$, he chose to perform the velocity averages in particle space rather than guiding center space. That is, he operated on the gyrokinetic equation with $J_{0}$ before evaluating the moment integrals. This approach has the advantage of easily reproducing the proper kinetic linear dispersion relation in an unsheared slab. The primary disadvantages lie in the complexity of the nonlinear and shear terms evaluated in particle space. 
Operating on Eq. (1) from the left with $J_{0}$, and keeping only the $E \times B$ nonlinearity, leads to

$$
\frac{\partial J_{0} F_{1}}{\partial t}+\nabla \cdot\left(v_{\|} J_{0}\left(F_{1} \hat{\mathbf{b}}\right)+F_{0} J_{0}^{2} \mathbf{v}_{E}+J_{0}\left(F_{1} J_{0} \mathbf{v}_{E}\right)\right)-\frac{\partial}{\partial v_{\|}}\left(\frac{e}{m} F_{0} J_{0}\left(\hat{\mathbf{b}} \cdot \nabla J_{0} \Phi\right)\right)=0 .
$$

We then take moments of this equation, using the definitions

$$
\bar{n}_{1} \equiv \int d^{3} v J_{0} F_{1}, \quad \bar{n}_{0} \bar{u}_{\| 1} \equiv \int d^{3} v J_{0} v_{\|} F_{1}
$$

and so on. Deferring the discussion of magnetic shear for the mcment (so that $\dot{b}=\dot{z}$ and $J_{0} \nabla_{\|}-$ $\nabla_{\|} J_{0}=0$ ), we may carry out the velocity space averages by assuming that the equilibrium $F_{0}$ is Maxwellian with density and temperature gradients in the $\dot{x}$ direction. With the normalizations given in Eqs. (53) and (54), we find the linear evolution equation for the particle-space perturbed density $\bar{n}$ :

$$
\frac{\partial \bar{n}}{\partial t}=-\nabla_{\|} \bar{u}_{\|}-\left[\Gamma_{0}+\eta_{i}\left(\Gamma_{0}-\Gamma_{1}\right) \nabla_{\perp}^{2}\right] \frac{\partial \Phi}{\partial y} .
$$

Note that by operating on the gyrokinetic equation with $J_{0}$ before taking velocity space integrals, we find terms $\propto \Gamma_{0,1}$ from integrals such as $\int d^{3} v F_{0} J_{0}^{2}$, and thus reproduce the proper kinetic FLR behavior, unlike the ", laxwellian total $F$ " model of the previous section. We will show in Sec. III.C. 3 how the guiding center approach is able to reproduce Eq. (30) with the appropriate transformations from guiding center quantities $n, T_{\perp}, u_{\|}, q_{\perp}, \ldots$ to particle quantities $\bar{n}, \bar{u}_{\|}, \ldots$.

Unfortunately, the nonlinear term in Eq. (29) is quite complicated, since the leftmost gyroaveraging operator $J_{0}$ now operates on both the distribution function and the electrostatic potential. and

$$
J_{0}\left(F_{1} J_{0} \mathbf{v}_{E}\right) \neq F_{1} J_{0}^{2} \mathbf{v}_{E}
$$

Similon approximated (on $\mathrm{p} .93$ of his dissertation) this term by assuming that the $v_{\perp}$ dependence of $F_{1}$ was proportional to $F_{0} J_{0}\left(k_{\perp} v_{\perp} / \Omega\right)$, allowing him to develop a simple fluid model of FLR effects in the nonlinear $\mathbf{E} \times \mathbf{B}$ terms. However, errors of order $k_{\perp}^{2} \rho^{2}$ are introduced by this approximation. As we show in the next section, we find that by taking fluid moments in guiding center space (delaying the second gyroaveraging operation to Poisson's equation) we can avoid having to take moments of quantities such as $J_{0}\left(F_{1} J_{0} \mathbf{v}_{E}\right)$, and thus regain $k_{\perp}^{2} \rho^{2}$ accuracy.

A second complication in the particle-space approach arises from the fact that the gyroaveraging operator $J_{0}$ and the parallel derivative operator $\hat{b}(\vec{x}) \cdot \nabla$ do not commute when the magnetic field is sheared. This introduces a gyro-averaging of $k_{\|}$which may be important for some modes, as pointed out by Bakshi et al. ${ }^{28}$ and Linsker. ${ }^{29}$ In the usual sheared slab model, where 
$\dot{b}(x)=\dot{z}+\dot{y} x / L_{s}$, one finds

$$
J_{0}(\dot{b} \cdot \nabla \Phi)=\dot{b} \cdot \nabla\left(J_{0} \Phi\right)+\left(\rho^{2} / 2 L_{s}\right) \partial^{2} \Phi / \partial x \partial y+O\left(k_{\perp}^{4} \rho^{4}\right),
$$

using the small $b$ approximation for $J_{0}$. The fastest-growing modes typically have $k_{\perp} \rho \sim 1 / 2$ and an average $k_{\|}$of order $k_{y} \rho / L_{t}$ so that the shear correction term is typically smaller than the lowest order $k_{\|}$term by a factor of order $L_{t} / L_{\boldsymbol{s}}$, which is usually small. However, as Linsker ${ }^{29}$ has shown, this gyro-averaged shear effect can be quite important for other modes, particularly for narrow modes localized near the rational surface, where $k_{\|} \rightarrow 0$. Since there are modes with significant growth rates which satisfy this criterion, it is necessary to treat the gyro-averaging of the shear as accurately as other FLR corrections.

Similon noted the difficulty presented by the shear and pointed out that the expression

$$
\int d^{3} v F_{0} J_{0} \dot{b} \cdot \nabla J_{0} \Phi=n_{0}\left\langle J_{0} \hat{\mathbf{b}} \cdot \nabla J_{0}\right\rangle_{0} \Phi=\Gamma_{0}^{1 / 2} \hat{b} \cdot \nabla \Gamma_{0}^{1 / 2} \Phi,
$$

remained valid in the presence of shear. [This is proved most easily in Fourier space where the $x$ in $\dot{b}(x)$ becomes $-i \partial / \partial k_{x}$.] Though he used this identity for moments of the last term in Eq. (29), Similon ignored the effects of shear when taking moments of the $J_{0}\left(\hat{\mathbf{b}} \cdot \nabla v_{\|} F_{1}\right)$ term.

Our approach is motivated in part by Similon's insights, though we improve upon his approach by taking moments of the gyrokinetic equation in guiding-center space, and also extend it to allow the presence of equilibrium and perturbed temperature gradients. We outline the details of our approach in the next two sections.

\section{Guiding Center Approach}

Equations 7-12 were derived by taking moments of the gyrokinetic equation directly, without first operating on it with a second $J_{0}$ as was done in the previous section. The transformation from guiding center fluid quantities to particle fluid quantities will be considered in the next section. Here we focus on how to approximate terms such as $\left\langle J_{0}\right\rangle$ and show how they lead to various linear and nonlinear FLR effects.

As an example, consider the following term from Eq. (7):

$$
\nabla \cdot\left(n\left\langle J_{0}\right\rangle \mathbf{v}_{E}\right)
$$

From Eq. (2), one sees that in general $\left\langle J_{0}\right\rangle$ is a function of all $v_{\perp}^{2 \ell}$ moments of the guiding center $F$. We will rnake the following closure approximation to express this in terms of just the lowest 
$\left\langle v_{\perp}^{2}\right\rangle=T_{\perp} / m$ moment, whose dynamics we do follow:

$$
\left\langle J_{0}\right\rangle \approx \Gamma_{0}^{1 / 2}(b)+N L P M
$$

where NLPM is a model of nonlinear, FLR-induced phase-mixing to be discussed in Section III.D. This $\Gamma_{0}^{1 / 2}(b)$ approximation was motivated by the contrast between the "Maxwellian total F" model which led to $\left\langle J_{0}\right\rangle=\exp (-b / 2)$, and Similon's real space approach where (at least linearly in a shearless slab) two Bessel functions are combined before taking moments, leading to $\left\langle J_{0}^{2}\right\rangle=\Gamma_{0}(b)$. In some sense, our $\left\langle J_{0}\right\rangle \approx\left\langle J_{0}^{2}\right\rangle^{1 / 2}=\Gamma_{0}^{1 / 2}(b)$ approximation anticipates the second gyroaveraging operation which will occur for the Poisson equation. Note that it is rigorous through second order in $k_{\perp} \rho$ (i.e., it matches the second-order Taylor series result $\left\langle J_{0}\right\rangle=1-b / 2$ ), and therefore agrees with the $\exp (-b / 2)$ model through second order, while imposing less gyroaveraging than the exponential model for large $b$. We will find that the $\Gamma_{0}^{1 / 2}$ model has the added advantage of exactly reproducing the FLR effects in the local, linear (unsheared slab) kinetic dispersion relation.

Both the equilibrium and fluctuating components of the perpendicular temperature are "hidden" in the argument to the Bessel function, since $b \propto T_{\perp 0}+T_{\perp 1}$. Using the chain rule to carry out the divergence, we find that

$$
\nabla \cdot\left(n \Gamma_{0}^{1 / 2}(b) \mathbf{v}_{E}\right)=(\nabla n) \cdot \Gamma_{0}^{1 / 2} \mathbf{v}_{E}+n(\nabla b) \frac{\partial \Gamma_{0}^{1 / 2}}{\partial b} \cdot \mathbf{v}_{E}+n \Gamma_{0}^{1 / 2}\left(\nabla \cdot \mathbf{v}_{E}\right)
$$

Although $T_{\perp 1} \ll T_{\perp 0}, \nabla_{\perp} T_{\perp 1} \sim \nabla_{\perp} T_{\perp 0}$. Thus, while $b$ may be replaced with $b_{0}$ in terms (1) and (3), the gradient in (3) produces two terms, proportional to $\nabla b_{0}$ and $\nabla b_{1}$. Because $\Gamma_{0}^{1 / 2}$ operates unambiguously on $\Phi$, we define $\Psi \equiv \Gamma_{0}^{1 / 2}\left(b_{0}\right) \Phi$ and $\mathbf{v}_{\Psi} \equiv \Gamma_{0}^{1 / 2}\left(b_{0}\right) \mathbf{v}_{E}$. For notational convenience, we now introduce two modified Laplacian operators $\dot{\nabla}_{\perp}^{2}$ and $\dot{\nabla}_{\perp}^{2}$ :

$$
\frac{1}{2} \dot{\nabla}_{\perp}^{2} \Gamma_{0}^{1 / 2} \equiv b \frac{\partial \Gamma_{0}^{1 / 2}}{\partial b} \quad \dot{\vec{\nabla}}_{\perp}^{2} \Gamma_{0}^{1 / 2} \equiv b \frac{\partial}{\partial b}\left(\Gamma_{0}^{1 / 2}+b \frac{\partial \Gamma_{0}^{1 / 2}}{\partial b}\right) .
$$

Note that as $b \rightarrow 0$, the operators $\dot{\nabla}_{\perp}^{2}, \dot{\nabla}_{\perp}^{2} \rightarrow \nabla_{L}^{2}$.

In slab geometry, the divergence of $\mathbf{v}_{E}$ vanishes from Eq. (33), leaving:

$$
\nabla \cdot\left(n \Gamma_{0}^{1 / 2}(b) \mathbf{v}_{E}\right)=\left(\frac{\partial n_{0}}{\partial x} \hat{x}+\nabla n_{1}\right) \cdot \mathbf{v}_{\Psi}+\frac{n_{0}}{T_{\perp 0}}\left(\frac{\partial T_{\perp 0}}{\partial x} \hat{x}+\nabla T_{\perp 1}\right) \cdot \frac{\hat{\nabla}_{1}^{2}}{2} \mathbf{v}_{\Psi}
$$

in which we have separated $n$ and $T_{\perp}$ into equilibrium and fluctuating parts as before. The last term on the right-hand side is a nonlinear FLR correction which appears quite naturally. In the small $k_{\perp} \rho$ limit, $\Gamma_{0}^{1 / 2}=1-k_{\perp}^{2} \rho^{2} / 2$ : these terms reduce to the familiar Taylor series expansion. 
However, in the large $k_{\perp} \rho$ limit, since $\dot{\nabla}_{\perp}^{2} \rightarrow-1 / 2$, this expression is much less stiff than the Taylor series expansion. Using the normalizations given in Eqs. (53) and (54), we find the equation for $\partial n / \partial t($ Eq. $(56))$.

Consider now the $\left\langle\left(m v_{\perp}^{2} / 2\right) J_{0}\right\rangle$ term which appears in the perpendicular pressure equation, Eq. (11). If the $v_{\perp}$ dependence of $F$ is close to Maxwellian (which is true at least for the linear $F_{0}$ component), then we can use the Maxwellian identity $\left(m v_{\perp}^{2} / 2\right) F_{m}=T_{\perp} \partial\left(T_{\perp} F_{m}\right) / \partial T_{\perp}$ to rewrite this in a form in which we can use Eq. (32):

$$
\left.n\left\langle\frac{m v_{\perp}^{2}}{2} J_{0}\right\rangle \approx T_{\perp} \frac{\partial}{\partial T_{\perp}}\left(T_{\perp} \int d^{3} v F J_{0}\right) \approx p_{\perp} \frac{\partial}{\partial b}\left(b \Gamma_{0}^{1 / 2}\right)\right)+N L P M .
$$

(Again, NLPM is a model of nonlinear, FLR-induced phase-mixing to be discussed in Sec. III.D.) In slab geometry, $\left\langle v_{\perp}^{2}\right\rangle$ enters only through FLR corrections, so to maintain overall second order accuracy it is only necessary to keep the lowest order contribution $\left\langle\left(m v_{\perp}^{2} / 2\right) J_{0}\right\rangle \approx T_{\perp}$. We use the more robust expression above to obtain higher order accuracy in the linear limit (where $F=F_{0}$ is Maxwellian) along with an approximate treatment of nonlinear effects consistent with the form of the closures used in other terms. [The situation in toroidal geometry is more complicated. There, the $\left\langle v_{\perp}^{2}\right\rangle$ moment enters through the grad B drifts as well, so that the number of $\left\langle v_{\perp}^{2 \ell}\right\rangle$ moments kept affects the accuracy of both the toroidal drift resonance model ${ }^{15,16}$ and the FLR model.] Inserting this expression for $\left\langle\left(m v_{\perp}^{2} / 2\right) J_{0}\right\rangle$ into Eq. (11), and expanding in the gyrokinetic ordering as we did for the density equation, we arrive at the linear and nonlinear FLR terms found in Eq. (60).

Finally, we should also explicitly state $t_{1} \omega w$ we approximate terms like $\left\langle v_{\|} J_{0}\right\rangle$ and $\left\langle v_{\|}^{3} J_{0}\right\rangle$. Our reasoning is as follows:

$$
\begin{aligned}
\left\langle v_{\|} J_{0}\right\rangle & =\left\langle\left(\left(v_{\|}-u_{\|}\right)+u_{\|}\right) J_{0}\right\rangle \\
& \approx\left(u_{\|}+q_{\perp} \frac{\hat{\nabla}_{\perp}^{2}}{2}\right) \Gamma_{0}^{1 / 2},
\end{aligned}
$$

where the heat flux $q_{\perp}$ results from the $\left\langle\left(v_{\|}-u_{\|}\right) v_{\perp}^{2}\right\rangle$ moment, and we have approximated the terms higher-order in $k_{\perp} \rho$ by analogy with Eq. (32). Similarly,

$$
\begin{aligned}
\left\langle v_{\|}^{3} J_{0}\right\rangle & =\left\langle\left(\left(v_{\|}-u_{\|}\right)^{3}+3 u_{\|} v_{\|}^{2}-3 u_{\|}^{2} v_{\|}+u_{\|}^{3}\right) J_{0}\right\rangle \\
& \approx\left(q_{\|}+3 u_{\|} p_{\|}\right) \Gamma_{0}^{1 / 2}+\text { higher order nonlinear terms. }
\end{aligned}
$$




\section{Guiding Center $\rightarrow$ Particle Space Transformation}

We have chosen to write our equations in guiding center space, in orde to take account of the gyroaveraging of the shear and to find the nonlinear terms most easily. It is not surprising to find that the simplicity we gained in the evolution equations is somewhat offset by a more complicated quasineutrality condition. We now develop a transformation from guiding center moments $\left(n, T_{1}, \ldots\right)$ to the particle moments $\left(\tilde{n}, \bar{u}_{\|}, \ldots\right)$. This is necessary because the Poisson equation. Eq. (3), depends directly on $\bar{n}$, not $n$. Along the way, we will also find that this transformation shows that particle fluid equations (such as Eq. (30)) are linearly equivalent to the guiding-center fluid equations, Eqs. (56-60).

The (non-polarization part of the) perturbed particle density is $\bar{n}_{1} \equiv \int d^{3} v J_{0} F_{1}$, as defined in Eq. (4). It is important to note that here, unlike in the evolution equations, the Bessel function operates on the distribution function.

We note that any linear solution of Eq. (1) in the absence of shear may be written as:

$$
\begin{gathered}
F_{1}\left(\mathbf{x}, v_{\|}, v_{\perp}, t\right)=F_{0}\left(\mathbf{x}, v_{\|}, v_{\perp}\right) J_{0}\left(\frac{v_{\perp}}{\Omega} k_{\perp}\right)\left[\alpha\left(\mathbf{x}, v_{\|}, t\right)+\left(\frac{m v_{\perp}^{2}}{2 T_{\perp 0}}-1\right) \beta\left(\mathbf{x}, v_{\|}, t\right)\right] \\
=F_{0} J_{0} \alpha+T_{\perp 0} \frac{\partial F_{0}}{\partial T_{\perp 0}} J_{0} \beta,
\end{gathered}
$$

in which $F_{0}$ is the equilibrium distribution function, $J_{0}$ operates on $\alpha$ aud $\beta$, and $\alpha$ and $\beta$ are functions independent of $v_{\perp}$. The moments of $\alpha$ and $\beta$ are determined self-consistently from the definitions of the guiding center fluid quantities

$$
\int d^{3} v F_{1} \equiv n \quad \int d^{3} v \frac{m v_{\perp}^{2}}{2} F_{1} \equiv p_{\perp} .
$$

Substituting Eq. (35) into Eqs. (36), and using the normalizations given in Eqs. (53) and (54), we find two equations in two unknowns:

$$
\begin{gathered}
n=\left\langle J_{0}\right\rangle\langle\alpha\rangle+b \frac{\partial\left\langle J_{0}\right\rangle}{\partial b}\langle\beta\rangle \\
p_{\perp}=\left[\left(1+b \frac{\partial}{\partial b}\right)\left\langle J_{0}\right\rangle\right]\langle\alpha\rangle+\left[\left(1+b \frac{\partial}{\partial b}+\frac{\partial}{\partial b}\left(b^{2} \frac{\partial}{\partial b}\right)\right)\left\langle J_{0}\right\rangle\right]\langle\beta\rangle
\end{gathered}
$$

Given the Ansat $z$ in Eq. (35), the transformation from the density of guiding centers to the density of particles is thus defined by $\left\langle J_{0}\right\rangle$ (Eq. (32)). The result of the algebra is

$$
\bar{n}=\frac{\Gamma_{0}^{1 / 2}}{D(b)}\left(N(b) n+\frac{1}{2} \dot{\nabla}_{\perp}^{2} T_{\perp}\right)
$$


in which

$$
N(b) \equiv 1+\dot{\nabla}_{\perp}^{2}-\frac{\dot{\nabla}_{\perp}^{4}}{2}
$$

and

$$
D(b) \equiv N(b)+\frac{\dot{\nabla}_{\perp}^{4}}{4}
$$

Note that $D(b)>0$. Expanding Eq. (37) for small $b$, one can verify that it reproduces Eq. (27) and is therefore second order accurate in $k_{\perp} \rho$. We may now find the guiding center quasineutrality condition (Eq. (62)) by noting that $n_{e 0}=n_{i 0}$, substituting Eq. (37) into Eq. (5), and operating on the resulting equation from the left with $\Gamma_{0}^{1 / 2}$.

In addition, we wish to ind an equation describing the evolution of $\bar{n}$. To accomplish this, we combine Eq. (37) and the linear parts of Eqs. (56) and (60), yielding:

$$
\frac{\partial \bar{n}}{\partial t}=-\frac{\Gamma_{0}^{1 / 2}}{D(b)} \nabla_{\|}\left(N(b) u_{\|}+\frac{1}{2} \dot{\nabla}_{\perp}^{2} q_{\perp}\right)-\left[\Gamma_{0}+\eta_{i}\left(\Gamma_{0}-\Gamma_{1}\right) \nabla_{\perp}^{2}\right] \frac{\partial \Phi}{\partial y} .
$$

Note that the coefficients of $\partial \Phi / \partial y$ in Eqs. (30) and (40) are exactly the same. A comparison of the parallel convection terms suggests that the transformation given in Eq. (37) may be generalized. This is the case, as the transformations

$$
\begin{aligned}
\bar{u}_{\|} & \equiv \frac{\Gamma_{0}^{1 / 2}}{D(b)}\left(N(b) u_{\|}+\frac{1}{2} \dot{\nabla}_{\perp}^{2} q_{\perp}\right) \\
\bar{p}_{\|} & \equiv \frac{\Gamma_{0}^{1 / 2}}{D(b)}\left(N(b) p_{\|}+\frac{1}{2} \dot{\nabla}_{\perp}^{2} r_{\perp}\right) \\
\bar{q}_{\|} & \equiv \frac{\Gamma_{0}^{1 / 2}}{D(b)}\left(N(b) q_{\|}+\frac{1}{2} \dot{\nabla}_{\perp}^{2} s_{\perp}\right)
\end{aligned}
$$

are consistent with the observation made in Section III.B that the parallel phase-mixing closure for perpendicular temperature fluctuations had the same form as the closure for parallel temperature fluctations (i.e., a 3 -moment closure for $p_{\|}$has the same form as the 3-moment closure for $r_{L}$, and can be combined by the above transformations to give the same form for a 3-moment closure for $\bar{p}_{\| 1}$ ) Ignoring the nonlinear terms, which are unfortunately very complicated in particle space, these transformations provide an alternate route to Eqs. (A5-A8) once Eqs. (56-59) are known. Specifically, one can find the linear $4+4$ gyrofluid model by repeating the procedure in Section III.C.1 for the parallel particle space moments $\bar{n}, \bar{u}_{\|}, \bar{T}_{\|}$and $\bar{q}_{\|}$, closing the resulting four equations as outlined in Ref. 1, and utilizing the transformations (Eqs. $(37,41-43)$ ) along with Eqs. (56-59). The moment-reduction scheme outlined in Sec. III.B.3 then uniquely determines the closures for models which retain fewer moments. While this gives one confidence that the linear 
FLR and parallel phase-mixing models have a degree of internal consistency, the complicated form of the nonlinear moments which arises in the particle space derivation precludes one from taking full advantage of this route. It is more straightforward to deveiop accurate models of nonlinear terms by taking moments in guiding center space.

\section{Alternative FLR Models}

It is perhaps not obvious why such an elaborate FLR model is necessary. Fig. 3 compares different approximations for $\left\langle j_{0}\right\rangle$. Clearly, though valid in the small $b$ limit, the Taylor series approximation is disastrously inaccurate for $b \geq 1$; other approximations are better. In particular, we have investigated the equations resulting from the $\left\langle J_{0}\right\rangle=e^{-b / 2},(1+b / 2)^{-1}$ approximations, described briefly below.

We may recover the sheared-slab, electrostatic limit of Brizard's gyrofluid equations ${ }^{20}$ from Eqs. (56-62) by leaving $q_{\|}$and $q_{\perp}$ unspecified, taking $\mathcal{N}_{1}=\mathcal{N}_{21}=\mathcal{N}_{22}=0, \Psi=e^{-b / 2} \Phi, N(b)=$ $D(b)=1$, redefining

$$
\frac{1}{2} \dot{\nabla}_{\perp}^{2} e^{-b / 2} \equiv b \frac{\partial e^{-b / 2}}{\partial b} \quad \dot{\bar{\nabla}}_{\perp}^{2} e^{-b / 2} \equiv b \frac{\partial}{\partial b}\left(e^{-b / 2}+b \frac{\partial e^{-b / 2}}{\partial b}\right)
$$

and replacing the $\Gamma_{0}$ in the " 7 " term of Eq. (62) with $e^{-b}$.

We have also considered a simpler FLR model which is second-order accurate, well-behaved at large $b$, and straightforward to implement in a finite-difference code. Though we solve Eqs. (56-62) with a spectral method which handles the expressions involving the $\Gamma_{0}^{1 / 2}$ functions easily, it may be advantageous for some problems to use the Pade approximation $\left\langle J_{0}\right\rangle=(1+b / 2)^{-1}$. We refer to this model as the "Padé" model for this reason, though it should be noted that the $\Gamma_{0}^{1 / 2}$ model is also a Padé-like approximation to the full FLR effects in the presence of magnetic shear. The Padé modei may be obtained from Eqs. (56-62) by taking $\Psi \equiv(1+b / 2)^{-1} \Phi, N(b)=D(b)=1$, redefining

$$
\frac{1}{2} \dot{\nabla}_{\perp}^{2}(1+b / 2)^{-1} \equiv b \frac{\partial(1+b / 2)^{-1}}{\partial b} \quad \dot{\nabla}_{\perp}^{2}(1+b / 2)^{-1} \equiv b \frac{\partial}{\partial b}\left((1+b / 2)^{-1}+b \frac{\partial(1+b / 2)^{-1}}{\partial b}\right) .
$$

replacing the $\Gamma_{0}$ in the " 7 " term of Eq. (62) with $(1+b / 2)^{-2}$ and the $\Gamma_{0}$ in the " 8 " term of Eq. (62) with $(1+b)^{-1}$. We present some results from this approach in Section V.A. 


\section{FLR Phase-Mixing}

The phase-mixing process which underlies Landau damping is fundamentally due to the distribution of velocities of the particles freely streaming along the field lines. This spread in velocities causes neighboring particles to move apart, mixing away (damping) any density perturbations which arise, even in the asymptotically collisionless limit. In addition to the spread in parallel velocities of particles, there is also a spread in the gyro-averaged $\mathbf{E} \times \mathbf{B}$ drift velocities, which leads to phase-mixing in the perpendicular direction. Physically, high energy particles with large gyroradii will have a slower $\mathbf{E} \times \mathbf{B}$ drift than low energy particles with small gyroradii; this spread in drift velocities leads to mixing. This process does not appear to be simply related to the usual stochastic perpendicuiar heating normally associated with large amplitude fluctuations. ${ }^{8,33-35}$ Nonlinear FLR phase-mixing is a complicated process which is difficult to reproduce accurately with fluid models, but the models we present below reproduce its essential qualitative features, providing a nonlinear damping process important at large $k_{\perp} \rho$ and mixing-length levels of $\Phi$.
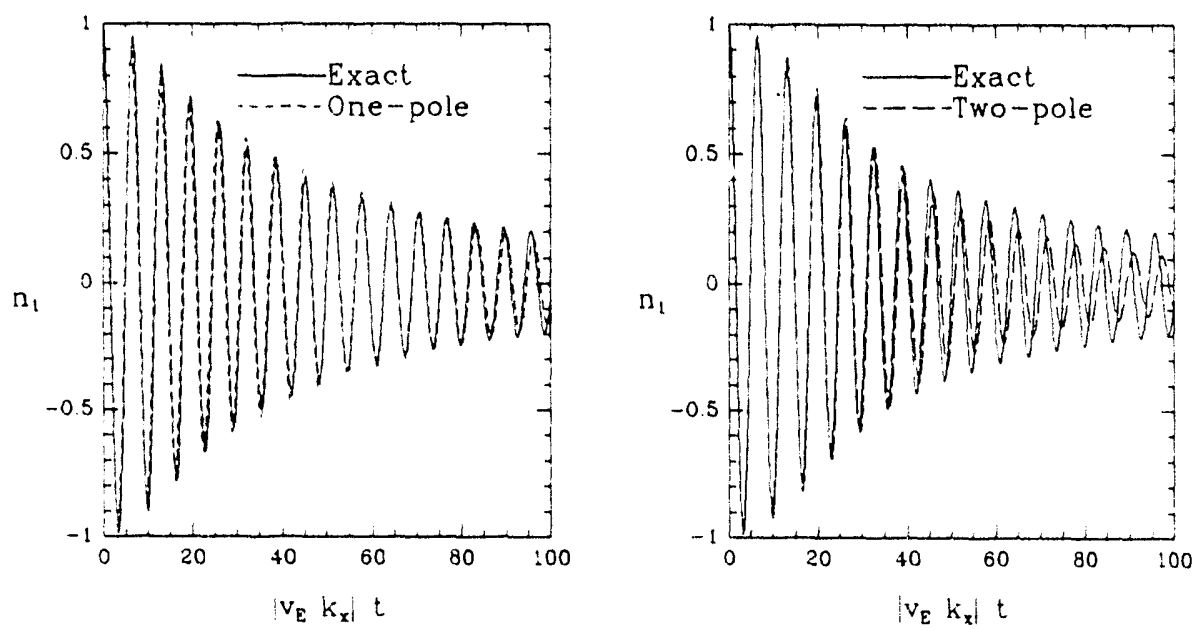

Figure 4: One- and two-pole FLR phase-mixing models, for $b=0.1$.

To illustrate the essence of this effect, we first consider a simplified 1-dimensional problem. where a given electrostatic field varies in only one direction (such as would be the case for a long, thin eddy. in the region away from one of the two regions of curvature). Taking $\Phi=\Phi(y)$, so $\mathbf{v}_{E}=v_{E}(y) x$, and assuming no gradients in the equilibrium $F_{0}$ and no parallel gradients, Eq. (1) reduces to

$$
\frac{\partial F_{1}}{\partial t}+J_{0}\left(\frac{k_{y} v_{\perp}}{\Omega}\right) v_{E} \frac{\partial F_{1}}{\partial x}=0
$$


We will concentrate on a single Fourier mode of $\Phi$, so the $k_{y}$ in $J_{0}$ can be considered a fixed number. We now look for the response of this equation to an initial pertubation of the form $F_{1}(t=0)=\exp \left(i k_{x} x\right) F_{m}(v)$, where $F_{m}$ is a Maxwellian. The solution is

$$
F_{1}=e^{i k_{x}\left(x-J_{0}\left(k_{y} v_{\perp} / \Omega\right) v_{E} t\right)} F_{m}(v) .
$$

Although $F_{1}$ oscillates in time, all velocity moments of $F_{1}$ will decay in time, just as in the parallel phase-mixing discussed earlier. For example, consider the density response

$$
n_{1 k}(t)=-i k_{x} x \int d^{3} v F_{1}=\int d^{3} v e^{-i k_{x} J_{0}\left(k_{y} v_{\perp} / \Omega\right) \mathbf{v}_{E} t} F_{m}(v) .
$$

Expanding $J_{0}$ in the small $k_{\perp} \rho$ limit, this integral can be evaluated analytically,

$$
\begin{aligned}
n_{1 k}(t) & \simeq \frac{n_{0}}{v_{t}^{2}} e^{-i k_{x} v_{E} t} \int_{0}^{\infty} d v_{\perp} v_{\perp} e^{i k_{x}\left(k_{y}^{2} v_{\perp}^{2} / 4 \Omega^{2}\right) v_{E} t} e^{-v_{\perp}^{2} /\left(2 v_{t}^{2}\right)} \\
& =n_{0} e^{-i k_{x} v_{E} t} \frac{1}{1-i k_{x} b v_{E} t / 2}
\end{aligned}
$$

where $b=k_{y}^{2} v_{t}^{2} / \Omega^{2}$ in this $1-D$ problem. [We could find an asymptotic representation of this integral without expanding in $k_{\perp} \rho$, using the method of stationary phase. However, the RiemannLebesgue lemma ${ }^{36}$ guarantees that the integral $\rightarrow 0$ as $t \rightarrow \infty$; since the fluid equations generate an exponentially damped approximation, which is too strong in any case, greater detail is unnecessary.] From Eq. (46), we see that the density decays in time, though it has a long tail $\sim 1 / t$, unlike the parallel phase-mixing case ${ }^{2}$ where initial density perturbations decay as $\exp \left(-k_{\|}^{2} v_{t}^{2} t^{2} / 2\right)$, faster than exponential. The full $J_{0}$ effects on $n_{1 k}(t)$ can be found by numerically integrating Eq. (45). The results are shown in Figs. 4-6. For $b>1$, there is a rapid initial decay followed by a long tail containing several harmonic components with slowly decaying amplitudes.

Because of the long tail, the time-Fourier transform of the kinetic response is not well-behaved as $\omega$ tends to zero, hence we are not able to match the kinetic and fluid results in frequency space as before. However, we may choose the closure coefficients so that the qualitative features of the true solution are preserved, and check our estimate numerically for a wide range of parameters.

\section{A 1-moment, 1-D Fluid Model}

The fluid model of parallel phase-mixing was based on a closure approximation for the $v_{\|} \nabla_{\|}$ kinetic term which introduced a damping rate of order

$$
\nu_{\| 1} \approx\left|k_{\|}\right| v_{t}=\left|k_{\|}\right|\left\langle\left(v_{\|}-\left\langle v_{\|}\right\rangle\right)^{2}\right\rangle^{1 / 2}
$$



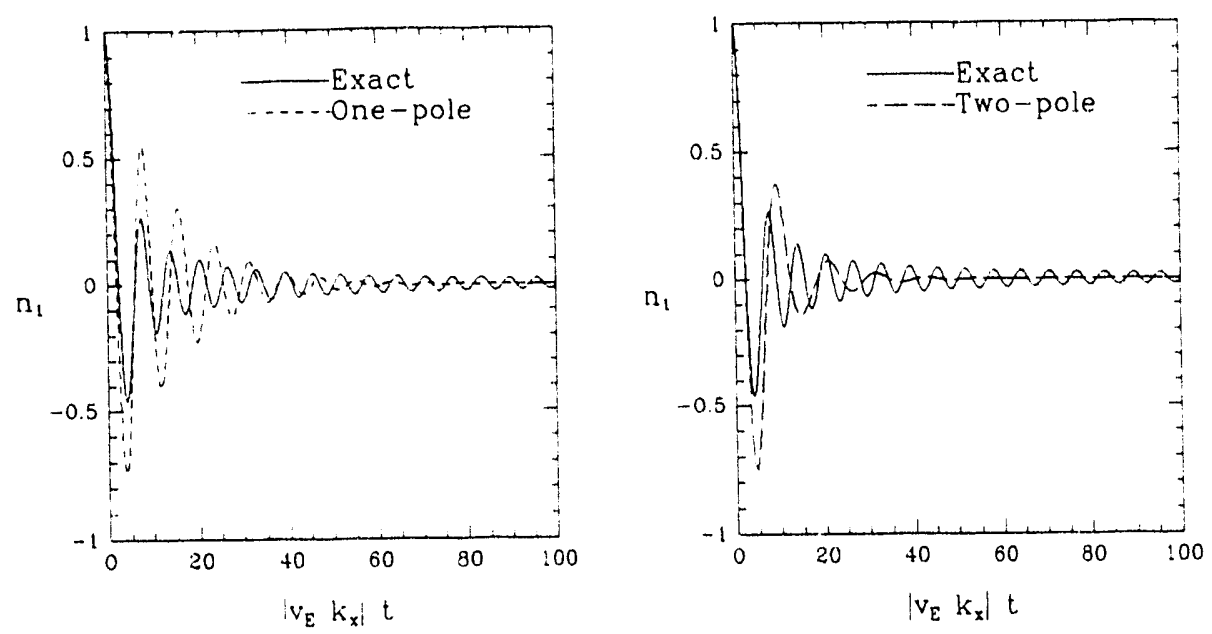

Figure 5: One- and two nole FLR phase-mixing models, for $b=1$.

Likewise, a fluid model of perpendicular FLR phase-mixing comes from making a closure approximation for the $J_{0} v_{E} \partial / \partial x$ term which introduces a damping term of order

$$
\nu_{\perp} \approx\left|k_{x}\right|\left\langle\left(J_{0} v_{E}-\left\langle J_{0} v_{E}\right\rangle\right)^{2}\right\rangle^{1 / 2}=\left|k_{x} v_{E}\right|\left(\Gamma_{0}(b)-e^{-b}\right)^{1 / 2},
$$

evaluating the averages $\langle\cdots\rangle$ over a Maxwellian. For small $k_{\perp} \rho$, this reduces to $\nu_{\perp} \approx\left|k_{x} v_{E}\right| b / 2$, which reproduces the initial decay rate in Eq. (46).
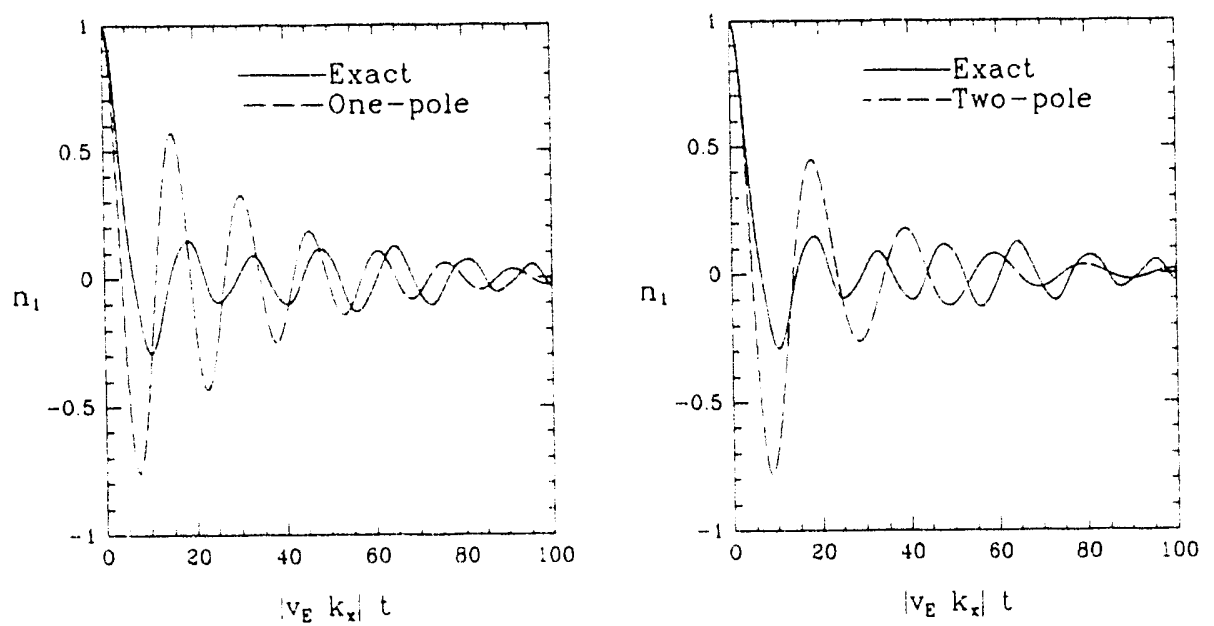

Figure 6: One- and two-pole FLR phase-mixing models, for $b=10$.

We will illustrate this by first developing a 1 -moment fluid model of FLR phase mixing. Taking 
the density moment of Eq. (45) gives

$$
\frac{\partial n}{\partial t}+\frac{\partial}{\partial x}\left(n\left\langle J_{0}\right\rangle v_{E}\right)=0
$$

Using a small $k_{y} \rho$ approximation for the Bessel function yields

$$
\frac{\partial n}{\partial t}+\left(1-\frac{b}{2}\right) v_{E} \frac{\partial n}{\partial x}-\frac{k_{x}^{2}}{2 m \Omega^{2}} n v_{E} \frac{\partial T_{\perp}}{\partial x}=0 .
$$

At this point we must introduce a closure approximation for the unknown highest moments $\left(T_{\perp}\right)$ in terms of the known lower moments $(n)$. If we were to neglect $\partial T_{\perp} / \partial x$, then we would just get $n(t)$ oscillating at a frequency $\omega=k_{x} v_{E}(1-b / 2)$ with no damping. For short times, this actually is similiar to the kinetic result $n_{1 k}(t) \approx \exp \left(-i k_{x} v_{E} t\right)\left(1+i k_{x} v_{E} b t / 2\right)+\cdots$, but it misses the long-time damped behavor of Eq. (46). To correct this, we follow the same procedure used for parallel closures and include a dissipative term in our a closure approximation for $T_{\perp}$,

$$
\frac{\partial n}{\partial t}+\left(1-\frac{b}{2}\right) v_{E} \frac{\partial n}{\partial x}=-\nu_{1} \frac{b}{2}\left|v_{E} k_{x}\right| n-\lambda_{1} \frac{b}{2} v_{E} \frac{\partial n}{\partial x} .
$$

The absolute value which appears in the first term on the right hand side guarantees that that term is always dissipative. The second (reactive) term allows us to match the phase of the fluid approximation to that of the kinetic solution. We expect the matching coefficients $\lambda_{1}$ and $\nu_{1}$ to be $O(1)$. Defining a relative squared error $\epsilon=\left(\int d t\left|n_{f}(t)-n_{1 k}(t)\right|^{2}\right) /\left(\int d t\left|n_{1 k}(t)\right|^{2}\right)$, where $n_{f}$ is the fluid approximation from Eq. (49) and $n_{1 k}$ is the kinetic result from Eq. (46), we find that a minimum value of $\epsilon=.17$ can be achieved with $\left(\nu_{1}, \lambda_{1}\right)=(0.4,0.6)$.

We extend this model to larger $k_{\perp} \rho$ by analogy with the results of Sec. III.C, replacing the $b$ 's which appear in the closure terms on the right hand side of Eq. (49) with the operator $-\dot{\nabla}_{\perp}^{2} \Gamma_{0}^{1 / 2}$. [The $(1-b / 2)$ factor on the left hand side becomes $\Gamma_{0}^{1 / 2}$ in accordance with Sec. III.C.] We define a one-pole FLR phase-mixing operator $\mathcal{N}_{1}$ operating on some moment $M$ by

$$
\mathcal{N}_{1} M=\nu_{1}\left|\left[\frac{1}{2} \dot{\nabla}_{\perp}^{2} \mathbf{v}_{\Psi}\right] \cdot \nabla\right| M-\lambda_{1}\left[\frac{1}{2} \dot{\nabla}_{\perp}^{2} \mathbf{v}_{\Psi}\right] \cdot \nabla M .
$$

where $\left(\nu_{1}, \lambda_{1}\right)=(0.4,0.6)$ as found in the small $b$ limit. The comparison between the 1 -moment fluid model employing this $\mathcal{N}_{1}$ operator, and the exact kinetic result from numerically integrating Eq. (45), is shown in Figs. 4-6. For small $b$, where the damping rate is small, the comparison is quite good. For $b$ of order 1 or larger, the agreement is not as good. Nevertheless, this is a significant improvement over past fluid models which ignored FLR phase-mixing altogether and would have oscillated in Figs. 4-6 without any damping. Some fluid models in the past employed 
an ad hoc linear hypervisocity term to provide damping at large $k_{\perp} \rho$. Our model provides a physics-based nonlinear damping term with at least the proper qualitative scaling with $\Phi, b$, and $k_{x}$.

\section{Extension to more moments and 2-D}

Rather than making a closure approximation for $T_{\perp}$ in Eq. (48), we can follow the dynamics for $T_{\perp}$ by taking the $v_{\perp}^{2}$ moment of Eq. (44). This requires a closure approximation for $\left\langle v_{\perp}^{2} J_{0}\right\rangle$, or at least of $\left\langle v_{\perp}^{4}\right\rangle$ if the small $k_{\perp} \rho$ expansion of $J_{0}$ is used. Following a similar path used to derive the 1-moment fluid model above, we finally arrive at the following two-moment model of FLR phase-mixing:

$$
\begin{aligned}
& \frac{d n}{d t}+\left[\frac{1}{2} \dot{\nabla}_{\perp}^{2} \mathbf{v}_{\Psi}\right] \cdot \nabla T_{\perp}+\mathcal{V}_{21} T_{\perp}=0 \\
& \frac{d T_{\perp}}{d t}+\left[\frac{1}{2} \dot{\nabla}_{\perp}^{2} \mathbf{v}_{\Psi}\right] \cdot \nabla n+\left[\dot{\nabla}_{\perp}^{2} \mathbf{v}_{\Psi}\right] \cdot \nabla T_{\perp}+\mathcal{N}_{22} T_{\perp}=0
\end{aligned}
$$

using the non-dimensional units of Eqs. (53-54), where $\mathcal{N}_{21}$ and $\mathcal{N}_{22}$ are given by:

$$
\begin{aligned}
& \mathcal{V}_{21}=-\frac{\nu_{2}}{4}\left|\left[\frac{1}{4} \dot{\nabla}_{\perp}^{4} \mathbf{v}_{\Psi}\right] \cdot \nabla\right|-\frac{\lambda_{2}}{4}\left[\frac{1}{4} \dot{\nabla}_{\perp}^{4} \mathbf{v}_{\Psi}\right] \cdot \nabla \\
& \mathcal{V}_{22}=\nu_{2}\left|\left[\frac{1}{2} \dot{\nabla}_{\perp}^{2} \mathbf{v}_{\Psi}\right] \cdot \nabla\right|-\lambda_{2}\left[\frac{1}{2} \dot{\nabla}_{\perp}^{2} \mathbf{v}_{\Psi}\right] \cdot \nabla .
\end{aligned}
$$

Choosing $\left(\nu_{2}, \lambda_{2}\right)=(1.6,1.3)$ gives a relative squared error in the small $b$ limit of $\varepsilon=\left(\int d t \mid n_{f}(t)-\right.$ $\left.\left.n_{1 k}(t)\right|^{2}\right) /\left(\int d t\left|n_{1 k}(t)\right|^{2}\right)=0.06$, somewhat better than the .17 achieved by the 1 -moment model. $\mathcal{V}_{21}$ represents closure approximations for higher order terms in $\left\langle J_{0}\right\rangle$ of order $k_{\perp}^{4} \rho^{4}$. A comparison with the numerical kinetic solution of Eq. (45) for general $b$ is shown in Figs. 4-6. Although the 2-moment model is formally more accurate than the 1 -moment model for small $b$, (and it recovers $\mathcal{O}(t)$ accuracy for the initial relaxation of a perturbation), we find that in practice it is of comparable accuracy to the 1-moment model for general $b$. An improved model employing additional moments could probably be found at the cost of additional complexity.

So far we have been considering the FLR phase-mixing caused by a given, static, 1-D, $\Phi(y)$. The kinetic equation is reversible, and if at some time $\Phi(y)$ suddenly changed sign (or if $\Phi=$ $\Phi(y) \cos \omega t$ was oscillatory), then $F_{1}$ would begin to "un-phase-mix" and eventually reconstruct the original density perturbation. [In fact, notice that our FLR phase-mixing model provides 
damping only nonlinearly and does not introduce any damping into the linear equations, since the linear $J_{0} \mathbf{v}_{E} \cdot \nabla F_{0}$ terms are known and do not require any closure approximations.] In a turbulent nonlinear 2-D system, it seems unlikely that the 2-D convection paths given by the equipotential lines of $J_{0} \Phi(x, y)$ would ever exactly reverse to reconstruct the initial perturbation. This is even less likely in a turbulent 3-D system where the $v_{\|}$of the particles would also have to be reversed to reconstruct the initial conditions. Nevertheless, the FLR phase-mixing process is very complicated, and it may be that in some cases (perhaps a weak-turbulence limit or a case involving a few isolated modes) our model may overestimate the amount of FLR phase-mixing which is actually occuring. In nonlinear simulations one should therefore check the decorrelation times and lengths, and also check the sensitivity of the simulations to the choice of $\nu$ and $\lambda$ coefficients in the $\mathcal{N}_{1}, \mathcal{N}_{21}$, and $\mathcal{V}_{22}$ operators.

Briefly, we outline one way to approximate terms like $\left|\dot{\nabla}_{\perp}^{2} \mathbf{v}_{E} \cdot \nabla\right| M$ in general geometry. First, note that $\dot{\nabla}_{\perp}^{2}$ operates only on $\mathbf{v}_{E}$, so we can define an averaged velocity field $\mathbf{v}=\hat{\nabla}_{\perp}^{2} \mathbf{v}_{E}$ using standard FFT's since the $\dot{\nabla}_{\perp}^{2}$ operator is evaluated most easily in $k$ space. Since this is already a crude model of a fairly complicated process, it may be sufficient to approximate

$$
|\mathbf{v} \cdot \nabla| \cdot M \simeq\left|v_{x}\right|\left|\frac{\partial}{\partial x}\right| M+\left|v_{y}\right|\left|\frac{\partial}{\partial y}\right| M,
$$

and then rearrange this to be in conservative form:

$$
|\mathbf{v} \cdot \nabla| M \simeq-\frac{\partial}{\partial x}\left(\left|v_{x}\right| \frac{1}{\left|k_{x}\right|} \frac{\partial M}{\partial x}\right)-\frac{\partial}{\partial y}\left(\left|v_{y}\right| \frac{1}{\left|k_{y}\right|} \frac{\partial M}{\partial y}\right) .
$$

The basic idea is to provide a fast numerical approximation by evaluating terms like $\left(1 /\left|k_{x}\right|\right) \partial M / \partial x$ in $k_{x}$ space where they are most easily evaluated, and then transform back to real space to multiply by $\left|v_{x}\right|$. This rough approximation will overestimate the FLR phase-mixing effect. since we have neglected "interference" terms inside the original absolute value. In the original kinetic equation, the $J_{0} \mathbf{v}_{E} \cdot \nabla F_{1}$ nonlinearity vanishes if both $\Phi$ and $F_{1}$ have cylindrical symmetry (or less restrictively, if $\left.\nabla_{\perp} F_{1} \| \nabla_{\perp} J_{0} \Phi\right)$, which may be related to the formation of long-lived coherent structures. The nonlinearity in Eq. (52) no longer satisfies this property exactly, though the nonlinear damping will be reduced for regions of the plasma which satisfy a kind of "square symmetry" where the two terms in Eq. (52) separately vanish. If a more accurate numerical implementation of $\mathcal{V M}$ is needed, one would probably have to map $M$ onto a grid which followed the convection contours, use FFT's along each convection contour to evaluate the phase-mixing from $|\mathbf{v}, \nabla| M$, and then map the phase-mixed.$M$ back to the regular grid where the rest of the terms in the fluid equations are advanced in time. 
As we have seen, FLR phase-mixing is a difficult process to model accurately with fluid equations. Hopefully the nonlinear simulations will not be too sensitive to the exact details of the damping mechanism at high $k_{\perp} \rho$, especially if the turbulent spectrum is peaked at longer wavelengths with $k_{\perp} \rho \ll 1$. Furthermore, the model we present here does capture the essential qualitative features of FLR phase-mixing with the proper physics-based scalings: a nonlinear damping process important at high $k_{\perp}$ and large $\Phi$.

\section{E. Normalized Gyrofluid Equations}

With the closure approximations specified, we may now complete the derivation of the nonlinear gyrofluid equations easily. The moments and the potential are separated into equilibrium and fluctuating components $\left(n=n_{0}+n_{1}(t), \ldots\right)$ and the normalizations used in Section III.C are utilized again. Specifically, the non-dimensional perturbed variables $\left(\tilde{n}_{1}, \bar{u}_{\|}, \ldots\right)$ are given in terms of the dimensional perturbed variables $\left(n_{1}, u_{\| 1}, \ldots\right)$ by

$$
\left(\frac{n_{1}}{n_{0}}, \frac{u_{\| 1}}{v_{t}}, \frac{T_{\| 1}}{T_{i}}, \frac{T_{\perp 1}}{T_{i}}, \frac{q_{\| 1}}{v_{t} p_{\| 0}}, \frac{q_{\perp 1}}{v_{t} p_{\perp 0}}, \frac{e \Phi_{1}}{T_{i}}\right)=\frac{\rho_{i}}{L_{n}}\left(\tilde{n}, \tilde{u}_{\|}, \tilde{T}_{\|}, \tilde{T}_{\perp}, \tilde{q}_{\| 1}, \tilde{q}_{\perp}, \dot{\Phi}\right) .
$$

Furthermore, we normalize $(x, y, z, t)$ according to

$$
\bar{x}=\frac{x-x_{0}}{\rho_{i}}, \quad \tilde{y}=\frac{y}{\rho_{i}}, \quad \bar{z}=\frac{z}{L_{n}}, \text { and } \tilde{t}=\frac{t v_{t}}{L_{n}} \text {. }
$$

We have used the definitions $L_{n}^{-1}=-\partial \ln n_{0} / \partial x, v_{t}=\sqrt{T_{0 i} / m_{i}}$ and $\rho_{i}=v_{t} / \Omega_{i}$. For convenience, we do not write the tildes over the non-dimensional variables except where confusion might otherwise be generated. Thus, throughout most of this paper, $\left(n, u_{\|}, \ldots\right)$ are the same as the non-dimensional variables $\left(\tilde{n}, \tilde{u}_{\|}, \ldots\right)$ in Eq. (53) and should not be confused with the original dimensional moments defined and used in Eq. (6)-12.

We set our model in the usual sheared-slab geometry. In dimensionless units, this leads to

$$
\begin{gathered}
\nabla_{L} \equiv \hat{\mathbf{x}} \frac{\partial}{\partial x}+\hat{\mathbf{y}} \frac{\partial}{\partial y} \quad \hat{\mathbf{b}} \cdot \nabla \equiv \frac{\partial}{\partial z}+s x \frac{\partial}{\partial y} \\
s \equiv L_{n} / L_{s}
\end{gathered}
$$

Denoting the gyroaveraged potential $\Gamma_{0}^{1 / 2}\left(b_{0}\right) \Phi \equiv \Psi$, we also define:

$$
\frac{d}{d t}=\frac{\partial}{\partial t}+\mathbf{v}_{\boldsymbol{\Psi}} \cdot \nabla, \quad \mathbf{v}_{\boldsymbol{\Psi}}=\dot{z} \times \nabla \Psi
$$

Neglecting the parallel nonlinearities (which are higher-order in $\Xi$ ) but retaining the $\mathrm{E} \times \mathrm{B}$ 
nonlinearities and the nonlinear FLR terms, we may obtain a $4+2$ gyrofluid model:

$$
\begin{aligned}
& \frac{d n}{d t}+\underbrace{\left[\frac{1}{2} \dot{\nabla}_{\perp}^{2} \mathbf{v}_{\Psi}\right] \cdot \nabla T_{\perp}}_{1}+\underbrace{V_{21} T_{\perp}}_{5}+\hat{\mathbf{b}} \cdot \nabla u_{\|}+\left[1+\eta_{i \perp} \frac{1}{2} \dot{\nabla}_{i}^{2}\right] \frac{\partial \Psi}{\partial y}=0 \\
& \frac{d u_{i 1}}{d t}+\underbrace{\left[\frac{1}{2} \nabla_{\perp}^{2} v_{\Psi}\right] \cdot \nabla q_{\perp}}_{1}+\underbrace{V_{21} q_{\perp}}_{5}+\hat{\mathbf{b}} \cdot \nabla\left(T_{11}+n+\Psi\right)=0 \\
& \frac{d T_{\|}}{d t}+\underbrace{V_{1} T_{\|}}_{5}+\hat{\mathbf{b}} \cdot \nabla\left(2 u_{\|}+q_{\|}\right)+\eta_{i \|} \frac{\partial \Psi}{\partial y}=0 \\
& \frac{d q_{\|}}{d t}-\underbrace{\left[\frac{3}{2} \dot{\nabla}_{\perp}^{2} v_{\Psi}\right] \cdot \nabla q_{\perp}}_{1}+\underbrace{V_{1} q_{\|}}_{5}+\hat{\mathbf{b}} \cdot \nabla(3+\underbrace{\beta_{\| 1}}_{2}) T_{\|}+\underbrace{\sqrt{2}\left|k_{\| 1}\right| D_{\|} q_{\|}}_{3}=0 \\
& \frac{d T_{\perp}}{d t}+\underbrace{\left[\frac{1}{2} \nabla_{\perp}^{2} \mathbf{v}_{\Psi}\right] \cdot \nabla n+\left[\dot{\nabla}_{\perp}^{2} \mathbf{v}_{\Psi}\right] \cdot \nabla T_{\perp}}_{1}+\underbrace{V_{22} T_{\perp}}_{;}+\hat{\mathbf{b}} \cdot \nabla q_{\perp}+\left[\frac{1}{2} \dot{\nabla}_{\perp}^{2}+\eta_{i_{\perp}}\left(1+\dot{\nabla}_{\perp}^{2}\right)\right] \frac{\partial \Psi}{\partial y}=0 \\
& \frac{d q_{\perp}}{d t}+\underbrace{\left[\frac{1}{2} \nabla_{\perp}^{2} \mathbf{v}_{\Psi}\right] \cdot \nabla u_{\|}+\left[\dot{\nabla}_{\perp}^{2} \mathbf{v}_{\Psi}\right] \cdot \nabla q_{\perp}}_{1}+\underbrace{V_{22} q_{\perp}}_{5}+\hat{\mathbf{b}} \cdot \nabla(\underbrace{\beta_{\perp} T_{\perp}}_{4}+\frac{1}{2} \dot{\nabla}_{\perp}^{2} \Psi)+\underbrace{\sqrt{2}\left|k_{\|}\right| D_{\perp} q_{\perp}}_{4}=0
\end{aligned}
$$

Poisson's equation (assuming quasineutrality and adiabatic electrons) is:

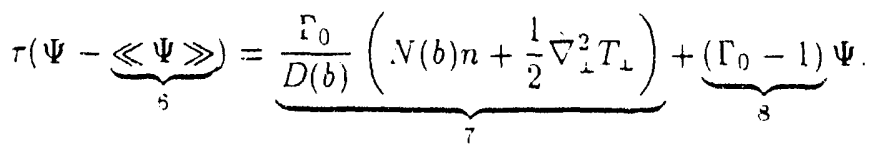

$T=T_{i 0} / T_{e 0}, \eta_{i}=L_{n} / L_{T}$, and $L_{T}^{-1}=-\partial \ln T_{0} / \partial x$. The argument of the Bessel functions is $b=\left(k_{x}^{2}+k_{y}^{2}\right)$. The modified perpendicular Laplacian operators $\nabla_{\perp}^{2}$ and $\dot{\nabla}_{\perp}^{2}$ are defined in Eqs. (34). The origins of the terms marked with underbraces are noted below.

(1) Vonlinear FLR terms which arise from the divergence of $\left\langle J_{0}\right\rangle$. The usual nonlinear terms are included in the definition of $d / d t$.

(2) The non-dissipative part of the $r_{\|}$closure: $\beta_{\|}=(32-9 \pi) /(3 \pi-8)$.

(3) The dissipative part of the $r_{\|}$closure; $D_{\|}=2 \sqrt{\pi} /(3 \pi-8)$.

(4) The $r_{-}$closure: $D_{\perp}=\sqrt{\pi} / 2$ and $\beta_{\perp}=1$

(5) A model of nonlinear FLR phase-mixing. The operators are defined in Eqs. (50) and (52). 
(6) In the absence of non-adiabatic electrons, the flux surface averaged potential must be explicitly subtracted to prevent non-physical electron transport across rational surfaces.

(7) With (8), the expression for the perturbed particle space density. $N(b)$ and $D(b)$ are defined in Eqs. (38) and (39).

(8) The usual ion polarization density.

A simpler (but still reasonably accurate) set of equations consists of evolution equations for the density, parallel velocity, and parallel and perpendicular temperatures (the " $3+1$ " model). In this model, the FLR phase-mixing of the parallel velocity is modeled with a one-pole model, rather than a two-pole model, and the closures for $q_{\|}$and $q_{\perp}$ are found by taking the $k_{\|} v_{t} \gg \omega, \omega$. limit of Eqs. (59) and (61) respectively. The result is:

$$
\begin{gathered}
\frac{d n}{d t}+\left[\frac{1}{2} \dot{\nabla}_{\perp}^{2} \mathbf{v}_{\Psi}\right] \cdot \nabla T_{\perp}+. V_{21} T_{\perp}+\hat{\mathrm{b}} \cdot \nabla u_{\|}+\left[1+\eta_{i \perp} \frac{1}{2} \dot{\nabla}_{\perp}^{2}\right] \frac{\partial \Psi}{\partial y}=0 \\
\frac{d u_{\|}}{d t}+\mathcal{V}_{1} u_{\|}+\hat{\mathrm{b}} \cdot \nabla\left(T_{\|}+n+\Psi\right)=0 \\
\frac{d T_{\|}}{d t}+\mathcal{N}_{1} T_{\|}+\hat{\mathrm{b}} \cdot \nabla 2 u_{\|}+\eta_{i \|} \frac{\partial \Psi}{\partial y}+\frac{3+\beta_{\|}}{\sqrt{2} D_{\|}}\left|k_{\|}\right| T_{\|}=0 \\
\frac{d T_{\perp}}{d t}+\left[\frac{1}{2} \nabla_{\perp}^{2} \mathbf{v}_{\Psi}\right] \cdot \nabla n+\left[\dot{\nabla}_{\perp}^{2} \mathbf{v}_{\Psi}\right] \cdot \nabla T_{\perp}+. \mathcal{V}_{22} T_{\perp} \\
+\left[\frac{1}{2} \dot{\nabla}_{\perp}^{2}+\eta_{i \perp}\left(1+\dot{\nabla}_{\perp}^{2}\right)\right] \frac{\partial \Psi}{\partial y}+\frac{1}{\sqrt{2} D_{\perp}}\left|k_{\|}\right|\left(\beta_{\perp} T_{\perp}+\frac{1}{2} \dot{\nabla}_{\perp}^{2} \Psi\right)=0
\end{gathered}
$$

Poisson's equation is not changed in this simpler model.

\section{The Local, Linear Dispersion Relation}

It is useful to consider the local limit as a partial check of our model. We could derive the local dispersion relation for the $4+2$ model by Fourier- and Laplace-transforming the linearized versions of Eqs. (56-62). The result is a polynomial in $\omega$ which may then be solved numerically. However. if equal numbers of parallel and perpendicular moments are kept, the dispersion relation may be factored into a form very similar to the familiar kinetic dispersion relation. For example, in dimensionless units, the dispersion relation for the $4+4$ model (see the Appendix) is

$$
1+\tau+\Gamma_{0}\left\{\left[1-\frac{k_{y} \tau}{\omega}\left(1-\eta_{i} b\left(1-\frac{I_{1}}{I_{0}}\right)\right)\right] \zeta Z_{4}(\zeta)-\frac{k_{y} \tau}{\omega} \eta_{i}\left[\zeta^{2}+\left(\zeta^{2}-\frac{1}{2}\right) \zeta Z_{4}(\zeta)\right]\right\}=0 .
$$


The only approximation to the usual kinetic result is $Z_{\mathbf{4}}(\zeta)$, given in Ref. 1. It is a four-pole approximation to the plasma dispersion function $Z(\zeta)$. In this limit, the $\Gamma_{0}^{1 / 2}$ FLR model is exact, to all orders in $k_{\perp} \rho$ for $n+n$ models [in the presence of temperature gradients, $n \geq 3$ ]. With the aid of .Mathematica ${ }^{37}$ and .Maple, ${ }^{38}$ we have derived the dispersion relations for each of the intermediate models (from $3+1$ to $4+4$ ). Except for the $n+n$ models, the dispersion relations cannot be easily factored into recognizable terms. Rather than delve into a lot of unpleasant algebra here, we will instead rely upon Figs. 7-9 to show a few important features of the local limit.
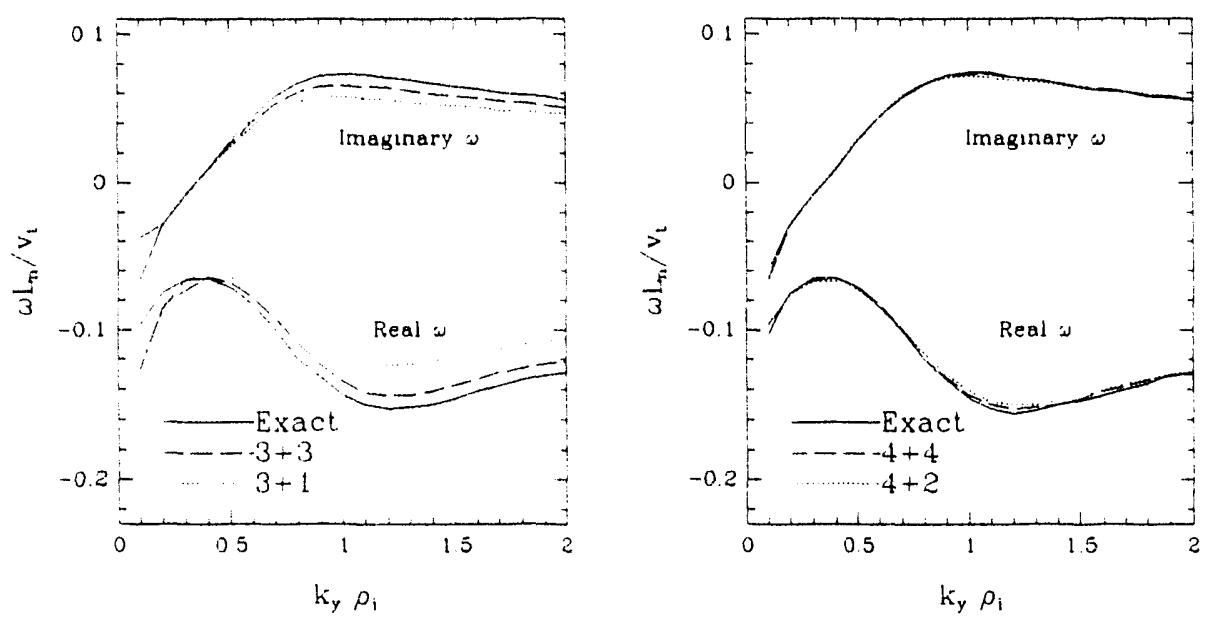

Figure $i$ : Local dispersion relation for $\eta_{i}=2, k_{\|} L_{n}=0.1$, and $T_{i}=T_{e}$.

In Fig. $7(a)$, the $3+3$ and $3+1$ models are compared to one branch of the local linear dispersion relation for $\eta_{i}=2, k_{11} L_{n}=0.1$, and $T_{i}=T_{e}$. Note that the agreement is good even for $k_{y} \rho_{i} \geq$ 1. where linear FLR effects are important. The improvement which made be obtained with additional moments is shown in Fig. $i(b)$. This kind of improvement, especially from the $3+1$ to the $4+2$ model, is quite typical of the local gyrofluid dispersion relations. Physically, it may be understood as follows: temperature fluctuations are quite important to the linear ITG dynamics, since the driving terms proportional to the background gradients appear in both the $\dot{T}_{\perp}$ and $\dot{T}_{\| 1}$ equations. Including the heat flux evolution equations provides two-pole models for the parallel and perpendicular temperature perturbations. Fig. 1 shows the improvement obtained as one moves from a one-pole model to a two-pole model of the expression $1+\zeta Z(\zeta)$. While this is not exactly the parallel temperature response function, the improvement in the corresponding 
expression is comparable.

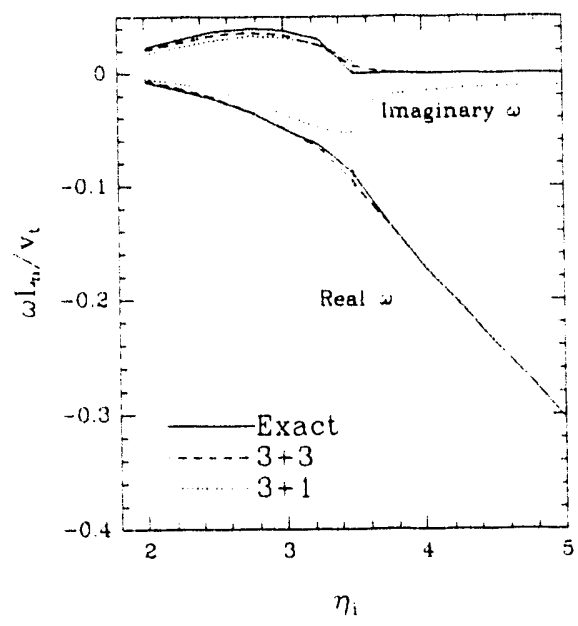

Figure 8: Local dispersion relation for $k_{\perp} \rho=.71, k_{\|} L_{n}=0.013$, and $T_{i}=T_{e}$.

Figure 8 compares the gyrofluid model to kinetic results shown in a previous paper. ${ }^{39}$ The parameters are $k_{\perp} \rho=.71, k_{\|} L_{n}=0.013$, and $T_{i}=T_{e}$. Above $\eta \simeq 3.4$, the kinetic analysis predicts no instability. The $3+3$ gyrofluid dispersion relation closely matches this kinetic result, since Eq. (67) differs from Eq. 11 of Dong et al. ${ }^{39}$ only in the plasma dispersion function. The limitations of the less accurate $3+1$ gyrofluid model are made more apparent. While the $3+1$ model reproduces the kinetic frequency with reasonable accuracy near $\eta_{i}=2$, the real frequency does not match as well as $\eta_{i}$ increases, and the second branch of the dispersion relation is overdamped. [For clarity, above $\eta_{i}=3.5$ we show the branch of the $3+1$ dispersion relation that corresponds to the marginally stable branch of the exact dispersion relation. There is another $3+1$ branch which remains very slightly unstable, with a different real frequency.] Based on this high- $k_{\perp} \rho$, low- $k_{\|} L_{n}$ example, we may expect to see the shortcomings of the $3+1$ model in the sheared system at high $k_{\perp} \rho$ near the rational surface. However, if we later find surprising results with the $3+1$ model, we may easily check them with a more accurate gyrofluid model.

From the local dispersion relation, we may also derive the marginal stability curve, $\eta_{i} v s . k_{y} \rho_{i}$. We are able to recover the kinetic result ${ }^{40,41}$ exactly using the $3+3$ or $4+4$ models, as shown in Figure 9. Also shown are marginal stability curves derived from the $3+1 \Gamma_{0}^{1 / 2}$ and $e^{-b / 2}$ models, and the commonly used, particle fluid Taylor series approximatiras to $\left\langle J_{0}^{2}\right\rangle$. The latter involves only three equations, for $\bar{n}, \bar{u}_{\| 1}$, and $\ddot{T}_{\|}$. For $k_{\perp} \rho=1$ the Taylur series curve crosses the $\eta$-axis. an 
unpinysical result.

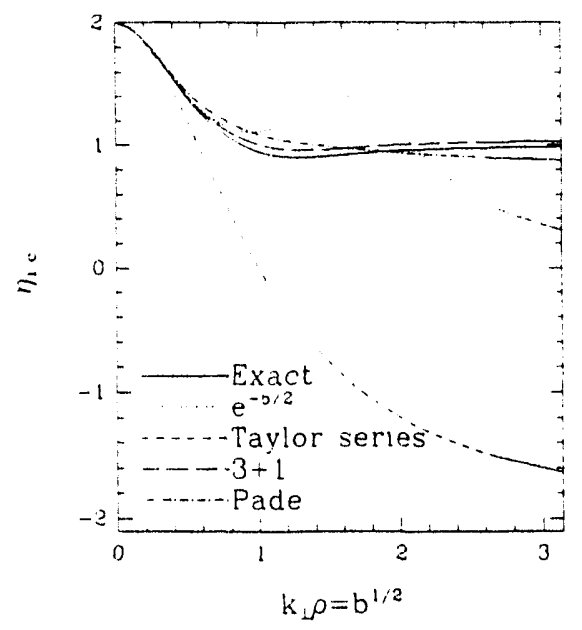

Figure 9: Marginal stability curves for different FLR models.

It is clear that the $\Gamma_{0}^{1 / 2}$ models more faithfully represent the linear physics than does the $e^{-b / 2}$ model. Given the roughly comparable complexity of the two models - they are equally demanding to implement numerically - there is no reason to choose the less accurate FLR model. The Padé approximation, on the other hand, represents a clear improvement over the Taylor series model, and is just slightly more difficult to implement in a finite-difference code, requiring only a standard tridiagonal solver.

\section{Linear Sheared Slab Results}

\section{A. Padé FLR Model}

While the Padé model (described in Sec. III.C.4) does not perform quite as well as the $\left\langle J_{0}\right\rangle=$ $\Gamma_{0}^{1 / 2}$ model in the large $k_{\perp} \rho$ limit (Fig. 9), it is nevertheless well-behaved, and has the advantage that it may be easily incorporated into existing finite-difference codes. Fig. 10 compares a linear eigenmode obtained with this model to the exact kinetic solution and to the commonly used particle space Taylor series result for a typical case. The parameters are $\eta_{i}=2, L_{s} / L_{n}=40.0, k_{y} \rho_{i}=0.6$ and $T_{i}=T_{e}$. The Taylor series approximations for the FLR effects predict a much wider mode than linear gyrokinetic theory. The gyrokinetic code finds $\omega=-.049+.039 i$, and the $4+2$ Pade model gives $\omega=-0.032+.032 i$. This level of error is typical of the Pade model. By way of 

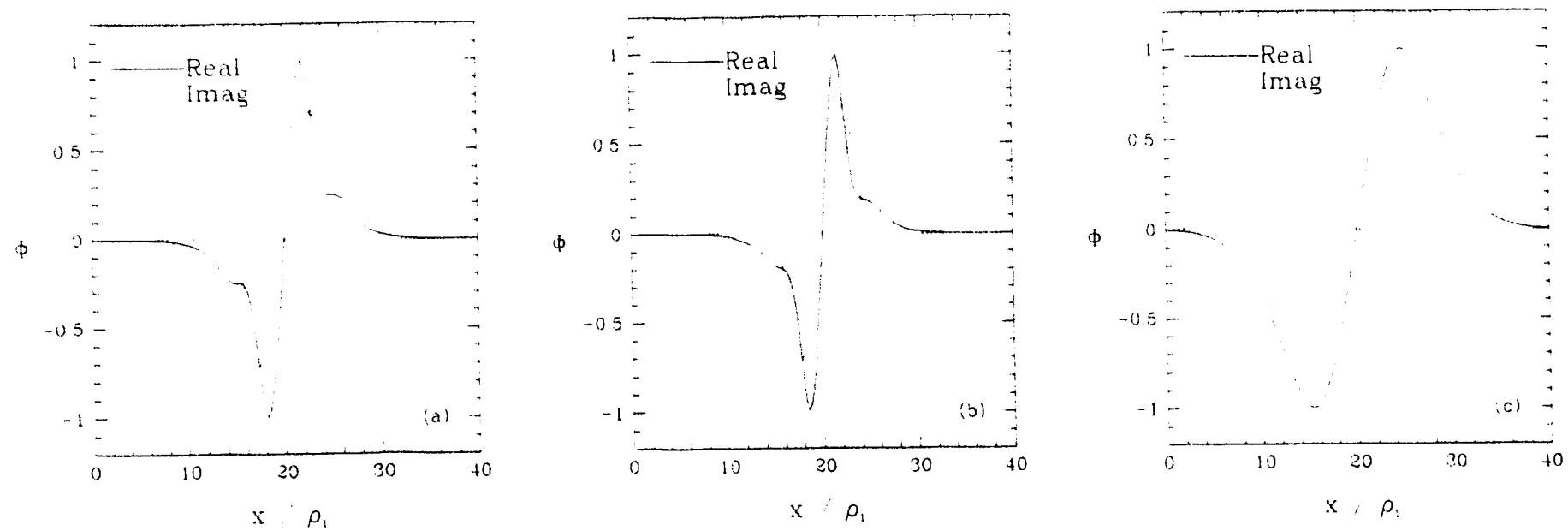

Figure 10: Electrostatic potential (arb. units) of the fastest-growing mode for $\eta_{i}=2, L_{s} / L_{n}=40$. $k_{y} \rho_{i}=0.6$ and $T_{i}=T_{e}$. (a) Gyrokinetic (b) Gyrofluid (Padé 4+2) (c) Taylor series.

comparison, the Taylor series model yields $\omega=-.170+.050 i$. While the Pade FLR model still misses the real frequency by $35 \%$, it is a substantial improvement over the Taylor series prediction. If simulations or analyses which include the full FLR effects show that the long-wavelength modes tend to dominate the saturated spectrum. the errors for the short wavelength modes may be tolerable it the $\left\langle J_{0}\right\rangle=(1+b / 2)^{-1}$ approximation.

\section{B. Full Kinetic Model}

Several years ago, Bakshi ${ }^{28}$ and Linsker ${ }^{29}$ pointed out a "kinematic term" related to the gyroaveraging of $k_{\|}$in systems with magnetic shear. All of the gyrofluid results presented below include this effect to second order in $k_{\perp} \rho$, sufficient accuracy to allow us to recover his Mode II. ${ }^{29}$ which he showed to exist only in the presence of the kinematic effect, and to be unstable for $k_{y} \rho_{i}>1$. We have found. however, that in practice, this effect is usually not significant for the majority of linear ITG eigenmodes, as the radial modifications do not extend beyond a very few gyroradii from the rational surface, and the frequency shifts are usually small.

Typical sheared-slab results from the $3+1$ model are shown in Fig. 11. The parameters are $\eta_{i}=2, L_{s} / L_{n}=40 . k_{y} f_{i}=0.707$ and $T_{i}=T_{i}$. The frequency found with Linsker's gyrokinetic integral code is $\omega=-.0674+.0414 i$; the $3+1$ gyrofluid model yields $\omega=-.0528+.0318 i$ Evolving the heat flux moments as well (the $t+2$ model) improves the frequency prediction to 

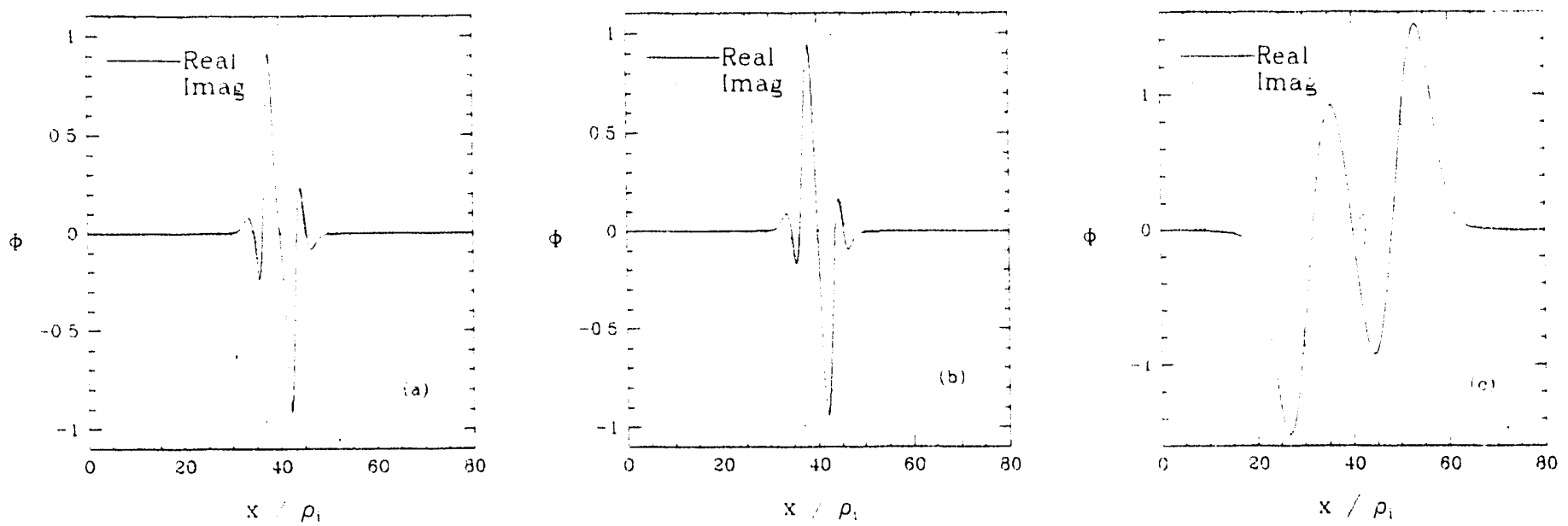

Figure 11: Electrostatic potential (arb. units) of the fastest-growing mode for $\eta_{i}=2, L_{s} / L_{n}=40$, $k_{y} \rho_{i}=0.707$ and $T_{i}=T_{e}$. (a) Gyrofluid result $(3+1)$ (b) Gyrokinetic result (c) Previous fluid model.

$\omega=-.06 i 4+.0384 i$. In Fig. 11(c) we show the result from a widely-used, particle space fluid model. The eigenmode is much broader than that found by either the gyrofluid or gyrokinetic codes, though qualitatively similar. The frequency predicted by this model, which uses a term $x \nabla_{\|\|}^{2}$ to model Landau damping and Taylor series expansions for the FLR effects, is $\omega=-.35+0.10 i$.

With this comparison, we clearly show that Landau damping and FLR effects can dramatically affect the dynamics of the ITG mode. We have found that both kinetic effects affect the linear ITG eigenmodes strongly, even in the small $k_{y} \rho$ limit or away irom marginal stability. In the latter case. since Landau damping is the only di pation in the system, the fastest-growing eigenmodes tend to arrange themselves so that the volume averaged fluid parameter $\left\langle\omega /\left(k_{\| \prime} v_{t}\right)\right\rangle$ is never large. A good FLR model is needed even in the long-wavelength limit, since as $k_{y} \rho_{i} \rightarrow 0$, the linear eigenmodes develop more radial structure, so that $b \sim 1$. This tendency may be noted in Figs. 12-13, as the unstable modes for small $k_{y} \rho_{i}$ tend to have the character of $l-3,4$.

Linear estimates of $\chi_{i}$. Often attention is focused on the $l=0$ (where $l$ is the radial mode number) mode in linear mixing-length studies. However, there is no physical or mathematical reason to exclude other eigenmodes from a mixing-length type estimate. particularly if the fastestgrowing or "largest" modes are not the $l=0$ modes. For most of the parameter-space in which 
we have benchmarked our code (low $\eta_{i}$, moderate to weak shear, and $\tau=1$ ), we find that other eigenmodes with comparable or larger average radial mode widths (despite the higher radial mode number) often have significantly larger growth rates thar the fundamental mode. Figs. 12-13 illustrate this point.

First. a note on how to interpret these figures is in order. Since we solve the gyrofluid equations as an initial-value problem, the resulting eigenmode corresponds to the fastest-growing mode, as long as the equations are evolved long enough to allow that mode to dominate. Thus, for each value of $k_{y} \rho_{i}$, the gyrofluid model yields a single solution. We have rot recovered the other branches of the dispersion relation by performing a Fourier transfcrm of the tume series data though tinis is certainly possible.

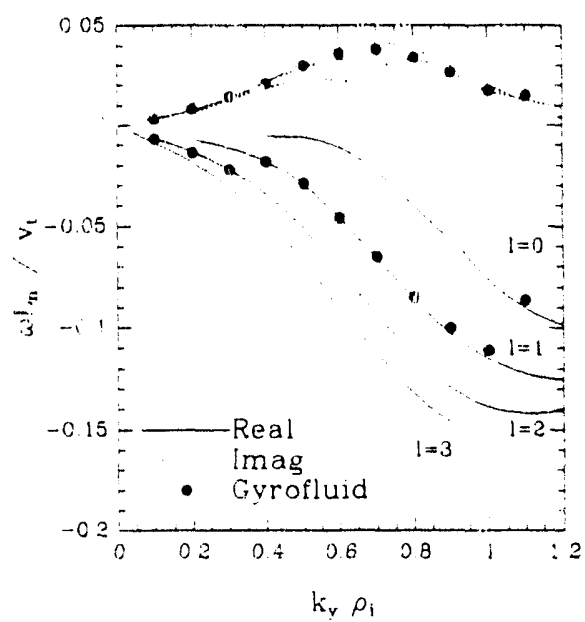

Figure 12: Linear dispersion relation for the first few radial eigenmodes for $\eta_{i}=2, L_{s} / L_{n}=40$. and $T_{l}=T_{0}$

Fig. 12 shows all of the branches of the linear dispersion relation for $\eta_{i}=2, L_{s} / L_{n}=40$, and $T_{i}=T_{\text {t }}$ which are at some point (For $0.1<k_{y} \rho_{i}<1.1$ ) fastest-growing for the $4+2$ gyrofluid model or the integral gyrokinetic code. ${ }^{29}$ The gyrofluid equations successfully resolve the mode with the largest growth rate in eacn case. The $l=0$ mode is not the fastest-growing mode until $k_{y} \rho_{i}>1$ : for most of the parameter space, the fastest-growing mode is of odd parity. with an $l=1$ character.

For $k_{y} \rho_{i}=0.7$, the fastest-growing mode in Fig. 12 is odd, with average radial mode width of 
$\Delta_{x}=1.9$, where :ve define the average radial mode width as

$$
\Delta_{x}^{2} \equiv \frac{\left\langle\Phi^{2}\right\rangle}{\left\langle\left(\frac{\partial \Phi}{\partial x}\right)^{2}\right\rangle}
$$

Here, the angle-brackets indicate a volume average. For the same parameters, the $l=0$ mode has $\Delta_{x}=1.4$. Given the additional difference in growth rates, a simple mixing-length estimate $\left(\gamma \Delta_{x}^{2}\right)$ of the transport from these two modes differs by a factor of three. At lower values of $k_{y} \rho_{i}$, for which the $l=0$ growth rate is much smaller, the discrepancy can be greater than an order of magnitude. Even worse, an analysis which focused only on the $l=0$ mode would incorrectly conclude that the system was only weakly unstable for $k_{y} \rho_{i} \leq 0.3$.
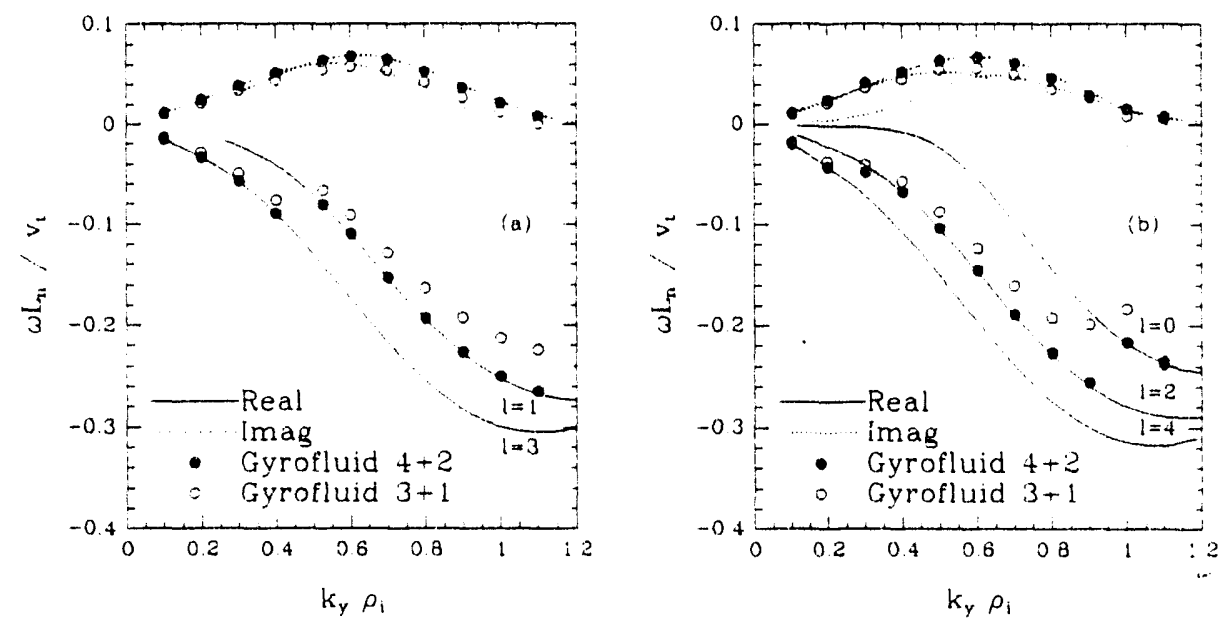

Figure 13: Linear dispersion relation for the first few radial eigenmodes for $\eta_{i}=3, L_{s} / L_{n}=40$, and $T_{i}=T_{e}$. (a) Odd parity (b) Even parity

Figure 13 shows the same information for the same parameters, except $\eta_{i}=3$ and the $3+1$ model is included. Also, the even and odd parity modes are shown on different panels for clarity. Again. the fastest-growing mode is odd for much of this region of parameter space, with the $l=0$ mode only becoming important at high $k_{y} \rho_{i}$. There is no single dominant radial eigenmode structure for the entire range of $k_{y} \rho_{i}$. Fig. 14(a) shows that the $l=0$ mode has the largest average radial mode width, yet the mixing length estimate of $x_{i}$ given by $\gamma /\left\langle k_{\perp}^{2}\right\rangle$ is largest for the $l=1$ mode for most values of $k_{y} \rho_{i}$ in this region of parameter space (Fig. 14(b)). Again, we emphasize that our gyrofluid model picks out the fastest-growing mode for each set of parameters; it should be clear that all of the radial eigenmodes are present from Figs. 12 and 13. 

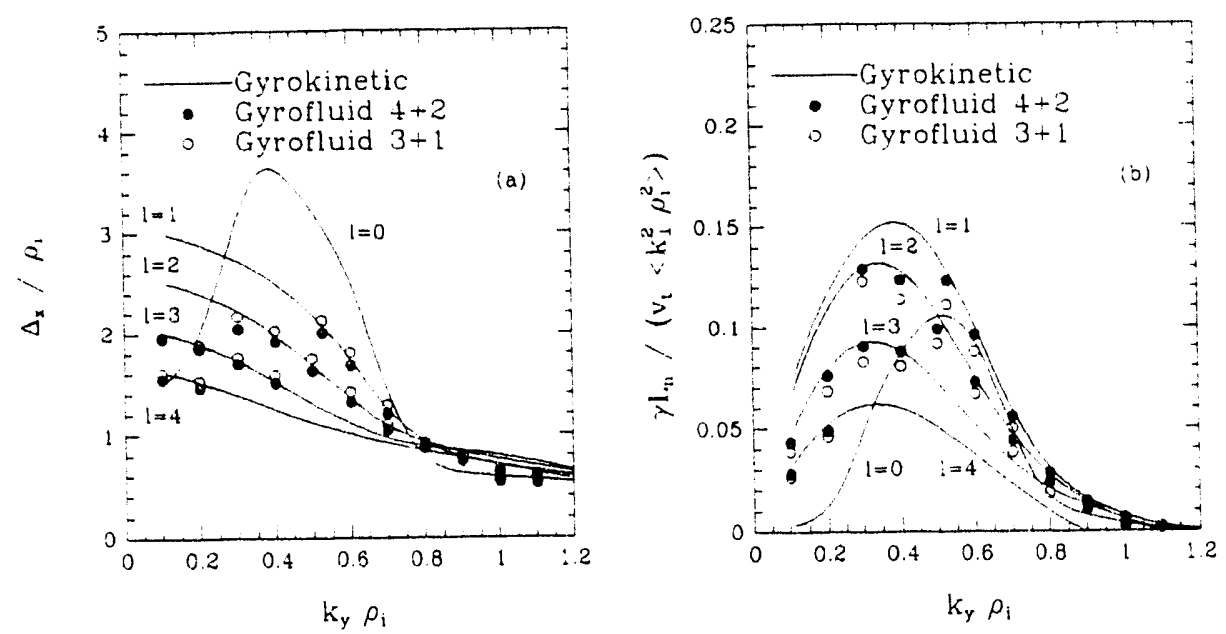

Figure 14: Mode widths and mixing length estimates for $\eta_{i}=3, L_{s} / L_{n}=40, k_{y} \rho_{i}=0.707$ and $T_{i}=T_{e}$. (a) Average mode width $\Delta_{x}$ (b) Mixing-length $\chi_{i}, \gamma /\left\langle k_{\perp}^{2}\right\rangle$

We also see from Figs. 13 and 14(b) that for these parameters, the peak of the mixing-length estimate of $\chi_{i}$ (at $k_{\perp} \rho_{i} \leq 0.4$ ) is downshifted $40 \%$ from the peak of the growth rate, which is at $k_{\perp} \rho_{i} \geq 0.6$ ). The downshift is more or less pronounced for different parameters, but indicate that the longer-wavelength modes are probably more important to transport than the simplest mixing-length arguments would suggest.

At least two others factors complicate a linear mixing-length estimate. First, there may be even longer wavelength modes which are linearly damped or weakly unstable yet which nevertheless play an important role in determining the nonlinear thermal transport. Second, fundamentally nonlinear processes ${ }^{11}$ may determine the relevant scales. Thus, we do not emphasize the mixing-length estimates here. We present them primarily to show that the gyrofluid equations compare very favorably with linear gyrokinetic theory, and to point out the potential hazards of concentrating one's linear analysis on a single unstable radial eigenmode. Having shown that our model performs satisfactorily in the linear regime, we may explore the nonlinear physics with more confidence.

\section{Conclusions}

Our goal has been to derive a fluid model which retains as much of the kinetic physics as possible, and to benchmark the equations and our numerical codes with the existing, well-developed 
linear gyrokinetic theory. We believe that our gyrofluid simulations will complement existing particle simulation efforts which are more fundamental and potentially more accurate. On a general note. we feel that our fluid approach, while certainly not appropriate for all problems, will be adequate for the investigation of tokamak turbulence, since (1) the nonlinear fluid equations express fundamental conservation laws which the turbulence must satisfy (conservation of particles, parallel momentum, parallel and perpendicular energy, and more if higher moments are retained), (2) they contain fairly accurate multipole models of the kinetic linear propagator, and are able to produce the proper linear frequency and wavenumber spectra, and (3) they contain the dominant nonlinearities ( $\mathrm{E} \times \mathrm{B}$ and the related FLR nonlinearities) which couple these linear modes together to provide the nonlinear saturation mechanism for the turbulent, nonlinear system.

We addressed several issues as we sought to extend the results of Ref. 1 from the shearless, drift-kinetic limit (their Eqs. (11ff)) to the sheared slab, gyrokinetic limit. First, we generalized the previous Landau damping closure to include new perpendicular velocity moment equations. We derived eight guiding center moment equations (for $n, u_{\|}, T_{\|}, q_{\|}, T_{\perp}, q_{\perp}, r_{\perp}$ and $s_{\perp}$ ) and showed how to reduce them to as few as may be needed for a particular application. We noted that for ITG turbulence, a $3+1$ model $\left(n, u_{\|}, T_{\|}\right.$and $\left.T_{\perp}\right)$ probably provides sufficient accuracy.

Second, we discussed how to approximate the velocity space averages of gyroaveraged quantities. We showed that the usual Taylor series approximations to these terms are inappropriate for numerical studies of ITG turbulence, as the radial grid spacing required to resolve the dynamics intrinsically involves modes with $k_{\perp} \rho>1$, for which the Taylor series approximations are inadequate. We presented two FLR models of varying complexity and utility, each second-order accurate in $k_{\perp} \rho$, and well-behaved for $k_{\perp} \rho \gg 1$.

Our most satisfactory model, the $\Gamma_{0}^{1 / 2}$ model, is linearly exact in the absence of magnetic shear if an equal number of parallel and perpendicular moments are kept. It can be easily implemented in existing spectral codes, as it requires only simple modifications of the perpendicular Laplacians and of Poisson's equation. These modifications also reduce the stiffness of the system, as the modified Laplacian operators are bounded by $\sim 0.5$ as $k_{\perp} \rho \rightarrow \infty$. With this FLR model in the gyrotluid equations, we match well the linear gyrokinetic predictions ${ }^{29,39}$ of frequencies, growth rates and mode structures even near marginal stabiiity, where the ITG mode is known to be strongly affected by kinetic effects.

We also outlined an FLR model more suitable for finite-difference applications, the Padé model. This model reproduces the kinetic growth rates to within only $\sim 25 \%$ accuracy in the regime in 
which we benchmarked its performance. However, we showed that with it. one may recover linear gyrokinetic eigenmodes with good accuracy. Like the $\Gamma_{0}^{1 / 2}$ model, the Pade model reduces the stiffness of the system.

Next, we described a new nonlinear, FLR phase-mixing effect. This perpendicular phasemixing process is analogous to parallel phase-mixing, deriving from the $\nabla \cdot\left(J_{0} \mathbf{v}_{E} F_{1}\right)$ term of Eq. (1), just as Landau damping derives from the $\nabla_{\|}\left(v_{\|} F_{1}\right)$ term. It may be as important as the Hasegawa-Mima ${ }^{9}$ polarization drift nonlinearity when $T_{i}=T_{e}$, as it is $\times k_{1}^{2}\left|\Phi_{\vec{k}} \vec{k} \times \overrightarrow{k^{\prime}}\right|$. It should provide an FLR-induced hyperviscosity-like sink of turbulent energy for $k_{\perp} \rho \geq 1$. We presented a gyrofluid model which captures the qualitative features of the resulting nonlinear gyrokinetic response. though we noted that it is a difficult effect to model accurately with fluid equations. We expect this effect to reduce the $\chi_{i}$ we e'ventually find with our simulations.

In carrying out the derivation of the new nonlinear gyrofluid terms, we showed a fairly general way to proceed when trying to model kinetic effects with fluid equations. For example, using the same method. one could find fluid models of the precessional drift resonance, cyclotron resonances, or the toroidal drift resonance. The latter has in fact already been accomplished. ${ }^{15,16}$ Additional simpler physics effects, such as non-adiabatic electrons and sheared equilibrium flows, have been added to this model with no complications; results will be reported in future publication.

Finally, we showed a few of the linear, numerical benchmarks we have carried out. Utilizing the kinetic models described above, we found excellent agreement with linear gyrokinetic theory. Previous fluid estimates of the linear frequencies and mode widths were generally too large by factors of $\sim 2$ and overestimated the nonlinear thermal flux by a factor of ten. ${ }^{27}$ From the evidence we presented here, we conclude that the $3+1, \Gamma_{0}^{1 / 2}$ model is probably sufficiently accurate for the study of ITG turbulence. In any event. we showed that our gyrofluid systems which retain more moments do become more accurate. Thus, we may (for example) ise the $4+2$ model to check nonlinear results obtained from the $3+1$ model for consistency.

In comparing with linear gyrokinetic theory, we pointed out that concentrating on a single radial eigenmode is likely to be misleading. We noted that mixing-length estimates can vary widely depending on which mode is selected, making such estimates uncertain unless a reliable selection criterion can be developed. Thus. we have begun nonlinear investigations of turbulent, nonlinear plasma phenomena numerically and analytically within this extended fluid paradigm to determine the scaling of $\chi_{i}$ with the nondimensional parameters of the problem. 


\section{Acknowledgments}

The authors thank M. A. Beer and J. V. W. Reynders for many useful discussions and for their help in benchmarking the gyrokinetic and gyrofluid codes, and S. Cowley for his insightful comments. We (in particular, WD) thank $\mathrm{M}$. Artun for helpful discussions about the gyrokinetic transformation and D. P. Coster and Q. P. Liu for their valuable computational experience and advice. The authors also thank the TFTR project for supporting this work which is ultimately aimed at developing quantitative tools for understanding tokamak turbulence. This work was supported by U. S. Department of Energy Contract No. DE-AC02-i6CH03073. 


\section{Appendix A. 4+4 Gyrofluid Model}

In this appendix, we present the nonlinear $4+4\left(n, u_{\|}, T_{\|}, q_{\|}, T_{\perp}, q_{\perp}, r_{\perp}\right.$ and $\left.s_{\perp}\right)$ gyrofluid model for completeness. While most problems probably do not require the accuracy afforded by this model, it is nevertheless useful to show that our closure schemes improve as more moments are retained. The nonlinear $4+4$ model equations are:

$$
\begin{aligned}
& \frac{d n}{d t}+\left[\frac{1}{2} \dot{\nabla}_{\perp}^{2} \mathbf{v}_{\Psi}\right] \cdot \nabla T_{\perp}+\dot{V}_{21} T_{\perp}+\hat{\mathrm{b}} \cdot \nabla u_{\|}+\left[1+\eta_{i \perp} \frac{1}{2} \dot{\nabla}_{\perp}^{2}\right] \frac{\partial \Psi}{\partial y}=0 \\
& \frac{d u_{\|}}{d t}+\left[\frac{1}{2} \dot{\nabla}_{\perp}^{2} \mathbf{v}_{\Psi}\right] \cdot \nabla q_{\perp}+v_{21} q_{\perp}+\hat{\mathbf{b}} \cdot \nabla\left(T_{\|}+n+\Psi\right)=0 \\
& \frac{d T_{\|}}{d t}+\mathcal{V}_{21} \mathcal{R}_{\perp}+\hat{\mathrm{b}} \cdot \nabla\left(2 u_{\|}+q_{\|}\right)+\eta_{i \|} \frac{\partial \Psi}{\partial y}=0 \\
& \frac{d q_{\|}}{d t}+. V_{21} s_{\perp}+\hat{\mathrm{b}} \cdot \nabla\left(3+\beta_{\|}\right) T_{\|}+\sqrt{2}\left|k_{\|}\right| D_{\|} q_{\|}=0 \\
& \frac{c^{\prime} T_{\perp}}{d t}+\left[\frac{1}{2} \nabla_{\perp}^{2} \mathbf{v}_{\Psi}\right] \cdot \nabla n+\left[\dot{\nabla}_{\perp}^{2} \mathbf{v}_{\Psi}\right] \cdot \nabla T_{\perp}+. V_{22} T_{\perp}+\hat{\mathrm{b}} \cdot \nabla q_{\perp}+\left[\frac{1}{2} \dot{\nabla}_{\perp}^{2}+\eta_{i \perp}\left(1+\dot{\nabla}_{\perp}^{2}\right)\right] \frac{\partial \Psi}{\partial y}=0 \\
& \frac{d q_{\perp}}{d t}+\left[\frac{1}{2} \dot{\nabla}_{\perp}^{2} \mathbf{v}_{\Psi}\right] \cdot \nabla u_{\|}+\left[\dot{\nabla}_{\perp}^{2} \mathbf{v}_{\Psi}\right] \cdot \nabla q_{\perp}+\dot{V}_{22} q_{\perp}+\hat{b} \cdot \nabla\left(\mathcal{R}_{\perp}+T_{\perp}+\frac{1}{2} \dot{\nabla}_{\perp}^{2} \Psi\right)=0 \\
& \frac{d \mathcal{R}_{\perp}}{d t}+\mathcal{V}_{22} r_{\perp}+\hat{\mathrm{b}} \cdot \nabla\left(2 q_{\perp}+s_{\perp}\right)+\eta_{i \|} \frac{1}{2} \nabla_{\perp}^{2} \frac{\partial \Psi}{\partial y}=0 \\
& \frac{d s_{\perp}}{d t}+\mathcal{N}_{22} s_{\perp}+\hat{\mathrm{b}} \cdot \nabla\left(3+\beta_{\|}\right) \mathcal{R}_{\perp}+\sqrt{2}\left|k_{\|}\right| D_{\|} s_{\perp}=0
\end{aligned}
$$

$R_{\perp}$ is defined by the relation:

$$
\mathcal{R}_{\perp} \equiv \frac{r_{\perp}}{T_{\perp}}
$$

(so that $\left.\mathcal{R}_{\perp 1}=r_{\perp 1}-T_{\perp 1}\right)$. Poisson's equation $(\mathrm{Eq} .(62)$ ) is unaffected by the additional moments. 


\section{References}

${ }^{1}$ G. W. Hammett and F. W. Perkins, Phys. Rev. Lett. 64, 3019 (1990).

${ }^{2}$ G. W. Hammett, W. Dorland, and F. W. Perkins, Phys. Fluids B 4, 2052 (1992).

${ }^{3}$ B. B. Kadomtsev and O. P. Pogutse, in Plasma Physics and Controlled Nuclear Fusion Research. 1984, volume 2, p. 69, International Atomic Energy Agency, Vienna, 1:385.

${ }^{4}$ Z. Chang and J. D. Callen, Phys. Fluids B 4, 1167 (1992).

${ }^{5} \mathrm{Z}$. Chang and J. D. Callen, Phys. Fluids B 4, 1182 (1992).

${ }^{5}$ A. Y. Aydemir, Phys. Fluids B 3, 3025 (1991).

${ }^{\top}$ R. D. Hazeltine, C. T. Hsu, and P. J. Morrison, Phys. Fluids 30, 3204 (1987)

${ }^{8}$ T. H. Dupree and D. J. Tetrault, Phys. Fluids 21, 425 (1978).

${ }^{9}$ A. Hasegawa and K. Mima, Phys. Rev. Lett. 39, 205 (1977).

${ }^{10}$ G. S. Lee and P. H. Diamond, Plıys. Fluids 29, 3291 (1986).

${ }^{11}$ S. C. Cowley, R. M. Kulsrud, and R. Sudan, Phys. Fluids B 3, 2767 (1991).

${ }^{12}$ E. A. Frieman and L. Chen, Phys. Fluids 25, 502 (1982).

${ }^{13}$ L. I. Rudakov and R. Z. Sagdeev, Dokl. Akad. Nauk. SSSR 138, 581 (1961).

${ }^{14}$ B. Coppi, M. N. Rosenbluth, and R. Z. Sagdeev, Phys. Fluids 10, 582 (1967).

${ }^{15}$ R. E. Waltz, R. R. Dominguez, and G. W. Hammett, Phys. Fluids B 4, 3138 (1992)

${ }^{16} \mathrm{M}$. A. Beer, private communication, 1992.

${ }^{17}$ S. Hamaguchi and W. Horton, Phys. Fluids B 2, 1833 (1990).

${ }^{18}$ S. Hamaguchi and W. Horton, Plasma Physics and Controlled Fusion 34, 203 (1992).

${ }^{19}$ W. W. Lee and W. M. Tang, Phys. Fluids 31, $612(1988)$

${ }^{20}$ A. Brizard, Phys. Fluids B 4, 1213 (1992).

${ }^{21} \mathrm{P}$. Similon, PhD thesis, Princeton University, 1981. 
${ }^{22}$ B. Lehnert, Plasma Physics and Controlled Fusion 31, 363 (1989).

${ }^{23}$ S. A. Smith, private communication, 1992.

${ }^{24}$ R. J. Hawryluk, V. Arunsalam, M. G. Bell, M. Bitter, W. R. Blanchard, N. L. Bretz, R. V. Budny, C. E. Bush, J. D. Callen, S. A. Cohen, S. K. Combs, S. L. Davis, D. L. Dimock, H. F. Dylla, P. C. Efthimion, L. C. Emerson, A. C. England, H. P. Eubank, R. J. Fonck, E. Frederickson, H. P. Furth, G. Gammel, R. J. Goldston, B. Grek, L. R. Grisham, G. W. Hammett, W. W. Heidbrink, H. W. Hendel, K. W. Hill, E. Hinnov, S. Hiroe, H. Hsuan, R. A. Hulse, K. P. Jaehnig, D. L. Jassby, F. C. Jobes, D. W. Johnson, L. C. Johnson, R. Kaita, R. Kamperschroer, S. M. Kaye, S. J. Kilpatrick, R. J. Knize, H. Kugel, P. H. LaMarche, B. LeBlanc, R. Little, C. H. Ma, D. M. Manos, D. K. Mansfield, R. T. McCann, M. P. McCarthy, D. C. McCune, K. McGuire, D. H. McNeill, D. M. Meade, S. S. Medley, D. R. Mikkelsen, S. L. Milora, W. Morris, D. Mueller, V. Mukhovatov, E. B. Nieschmidt, J. O'Rourke, D. K. Owens, H. Park, N. Pomphrey, B. Prichard, A. T. Ramsey, M. H. Redi, A. L. Roquemore, P. H. Rutherford, N. R. Sauthoff, G. Schilling, J. Schivell, G. L. Schmidt, S. D. Scott, S. Sesnic, J. C. Sinnis, F. T. Stauffer, B. C. Stratton, G. D. Tait, G. Taylor, J. R. Timberlake, H. H. Towner, M. Ulrickson, V. Vershkov, S. V. Goeler, F. Wagner, R. Wieland, J. B. Wilgen, M. Williams, K. L. Wong, S. Yoshikawa, R. Yoshino, K. M. Young, M. C. Zarnstorff, V. S. Zaveryaev, and S. J. Zweben, in Plasma Physics and Controlled Vuclear Fusion Research, 1986. volume 1, p. 51, International Atomic Energy Agency, Vienna, 1987.

${ }^{25}$ M. C. Zarnstorff, C. W. Barnes, P. C. Efthimion, G. W. Hammett, W. Horton, R. A. Hulse, D. K. Mansfield, E. S. Marmar, K. M. MícGuire, G. Rewoldt, B. C. Stratton, E. J. Synakowski, W. M. Tang, J. L. Terry, X. Q. Xu, M. G. Bell, M. Bitter, N. L. Bretz, R. V. Budny, C. E. Bush, P. H. Diamond, R. J. Fonck, E. D. Frederickson, H. P. Furth, R. J. Goldston, B. Grek, R. H. Hawryluk, K. W. Hill, H. Hsuan, D. W. Johnson, D. C. McCune, D. M. Meade, D. Mueller, D. K. Owens, H. K. Park, A. T. Ramsey, M. N. Rosenbluth, J. Schivell, G. L. Schmidt, S. D. Scott, G. Taylor, and R. M. Wieland, in Plasma Physics and Controlled Nuclear Fusion Research, 1990, volume 1, p. 109, International Atomic Energy Agency, Vienna, 1991.

${ }^{26}$ W. Horton, D. Lindberg, J. Y. Kim, J. Q. Dong, G. W. Hammett, S. D. Scott, and M. C. Zarnstorff, Phys. Fluids B 4, 953 (1992). 
${ }^{27}$ M. Kotschenreuther, H. L. Berk, R. Denton, S. Hamaguchi, W. Horton, C.-B. Kim. M. LeBrun, P. Lysier, S. Mahajan, W. H. Miner, P. J. Morrison, D. Ross, T. Tajima, J. B. Taylor, P. M. Valanju, H. V. Wong, S. Y. Xiao, and Y.-Z. Zhang, in Plasma Physics and Controlled Vuclear Fusıon Research, 1990, volume 2, p. 361, International Atomic Energy Agency, Vienna, 1991.

${ }^{28}$ See the references in Ref. 29.

${ }^{29}$ R. Linsker. Phys. Fluids 24, $1485(1981)$.

${ }^{30}$ W. W. Lee, Phys. Fluids 26, 556 (1983).

${ }^{31}$ W. W. Lee. J. Comput. Phys. 72, 243 (1987).

${ }^{32}$ T. S. Hahm, W. W. Lee, and A. Brizard, Phys. Fluids 31, 1940 (1988).

${ }^{33}$ J. A. Krommes, Phys. Fluids 23, 736 (1980).

${ }^{34}$ H. C. Ching, Phys. Fluids 16, 130 (1973).

${ }^{35}$ S. P. Hirshman, Phys. Fluids 23, 562 (1980).

${ }^{36}$ C. M. Bender and S. A. Orszag, Advanced Mathematical Methods for Scientists and Engineers, McGraw-Hill Book Company, New York, 1978.

${ }^{37}$ S. Wolfram, Wathematica, A System for Doing Mathematics by Computer, Addison-Wesley Publishing Co., New York, 1988.

${ }^{38}$ B. W. Char, K. O. Geddes, G. H. Gonnet, B. L. Leong, M. B. Monagan, and S. M. Watt, Maple $V$ Language Reference Manual, Springer-Verlag, Toronto, 1991.

${ }^{39}$ J. Q. Dong, P. N Guzdar, and Y. C. Lee, Phys. Fluids 30, 2694 (1987).

${ }^{40}$ A. A. Galeev. V. N. Oraevskii, and R. Z. Sagdeev, Soviet Physics JETP 17, 615 (1963).

${ }^{41}$ B. B. Kadomtsev and O. P. Pogutse, in Review of Plasma Physics, edited by M. A. Leontovich, Consultants Bureau, New York, 1970, p. 249, Vol. 2, No. 69. 
Dr. F. Proloni, Univ. of Wollongong, AUSTRALIA

Prof. M.H. Brennan, Univ. of Sydney, AUSTRALIA

Plasma Research Lab., Australian NaL. Univ., AUSTRALIA

Prot. I.R. Jonos, Flinders Univ, AUSTRALIA

Prof. F. Cap, Inst. for Theoretical Physics, AUSTRIA

Prof. M. Heindter, Institut for Theoretische Physik, AUSTRIA

Prof. M. Goossens, Astronomisch instituut, BELGIUM

Ecole Royado Militairs, Lob. do Phy. Plasmas, BELGIUM

Commission-Europeen, DG. XII-Fusion Prog., BELGIUM

Prof. R. Bouciqu, Rijkeuniveriteit Gent, BELGIUM

Dr. P.H. Sekanakn, Instituto Fieica, BRAZIL.

Intwitut Neciond De Pesquiens Especieie-INPE, BRLIL

Document Ofice, Atomic Energy of Cenada Ud., CANADA

Dr. M.P. Bechynekj, MPB Technologies, Inc., CANADA

Dr. H.M. Skareaged, Univ. of Sackatchowen, CANADA

Prof. J. Teichmam, Univ. of Montreal, CANADA

Prot. S.R. Sreanivasen, Univ. of Celogy, CANADA

Prof. T.W. Johnston, INAS-Energiv, CANADA

Dr. R. Bction, Centre canadien de fusion meonotique, CANADA

Dr. C.R. James., Univ. of Nberta, CANADA

Dr. P. Luktc, Komencketho Universzita, CZECHOSSLOVAKIA

The Librarian, Cuthem Laboratory, ENGLAND

Librey, RS1, Ruthartord Appleson Laboratory, ENGLANO

Nrs. S.A. Hutchinson, JET Librery, ENGLAND

Dr. S.C. Shamn, Univ. of South Prafic, FIJI ISLANDS

P. Mehonen, Univ. of Helsiniki, FINLAND

Prot. M.N. Buscec, Ecolo Potytechnique, FRANCE

C. Mouttor, Lab. de Physique des Milioux lonises, FRANCE

J. Radot, CENCADARACHE - Bat 506, FRANCE

Prof. E. Economou, Univ. ot Crete, GREECE

Ms. C. Rinni, Univ. of loamina, GREECE

Dr. T. Mud, Acedemy Bibliographic Ser., HONG KONG

Preprint Library, Hungarien Acadamy of Sci., HUNGARY

Dr. 8. DasGupta, Saha inst of Nucteer Ptyrics, INDIA

Dr. P. Kaw, Inst. for Plasma Research, INDIA

Dr. P. Roseneu, Iered Inst of Tectnology, ISRAEL

Librarian, Intomational Center for Theo Physics, ITALY

Miss C. De Pab, Associazione EURATOMENEA, ITALY

Dr. G. Groseo, letitute of Fisica dat Plasma. ITALY

Prof. G. Rostangni, Istituto Ges Ionizzati Dol Cnr, ITALY

Dr. H. Yamato, Tochiba Ros a Deval Center, JAPAN
Prot. I. Kewakemi, Hiroshima Univ., JAPAN

Prot. K. Nishikawa, Hiroshima Univ., JAPAN

Director, Jepen Atomic Energy Research inst., JAPAN

Prof. S. Itoh, Kyushu Univ., JAPAN

Research Into. Cr., National Instit. for Fusion Science. JAPAN

Prot. S. Tanake, Kyow Univ., JAPAN

Librery, Kyolo Univ., JAPAN

Prof. N. Inowe, Univ. of Tokyo, MAPAN

Secretary, Plesma Section, Electrotachnical Lab., JAPAN

S. Mori, Techniced Actieor, MEAI, LAPAN

Dr. O. Nitere, Kumenos Inst of Technology, JAPAN

J. Hyoon-Sook, Korea Atomic Enorgy Rosearch inst, KOREA

D.I. Choi, The Kore Adv. Inst of Sa. \& Tech., KOREA

Prof. B.S. Liby, Univ. of Waikato, NEW ZEALAND

Inst of Phytics, Chinese Aced Sci PEOPLE'S REP. OF CHINA

Librey, Inst of Plasma Physics, PEOPLE'S REP. OF CHINA

Tringhua Univ. Library, PEOPLE'S REPUBLIC OF CHINA

Z. Li, S.W. Inst Phyeics, PEOPLE'S REPUBLC OF CHINA

Prof. J.A.C. Cebre, Instuto Superior Tecnico, PORTUGAL

or. O. Potrus, al I Cuza Univ., Romania

Or. J. de Viliers, Fusion Studies, AEC, S. AFPICA

Prof. M.A. Helberv, Univ. of Natal, S. AFRICA

Prof. D.E. Km, Pohang Inst. of Sa. Tech., SO. KOREA

Prot. C.I.E.M.A.T. Fusion Division Libray, SPAIN

Dr. L Sanfio, Univ. of UMEA, SWEDEN

Libren, Royd inst. of Technology, SWEDEN

Prof. H. Whoimeon, Cheiman Univ. of Tech., SWEDEN

Conte Phys. Des Plaemas, Ecole Polytech, SWITZERLAND

Bibliotheek, Inat. Voor Plasma-Fysica, THE NETHERLANDS

Asst. Prot. Dr. S. Cakir, Midde East Tech. Univ., TURKEY

Dr. V.A. Guthiloh,Sci. Res. Inst Eloctrophys.I Apparatus, USSR

Dr. D.D. Ayutov, Siberien Brench of Academy of Sa., USSR

Dr. G.A. Eliceer, I.V. Kurchatov Inse, USSA

Librerien, The Ukr.SSA Acedemy of Scionces, USSR

Dr. L.M. Kovizhmykh, Inst. of Gonoral Physics, USSR

Kemtorectungeaniege GmbH, Zentrabibliothek, W. GERMANY

Bibliothak, inst. FOr Plasmatorechung, W. GERMANY

Prof. K. Schinder, Ruhr-Universitt Bochum, W. GEPMANY

Dr. F. Wogner, (ASDEX), Wax-Planck-Institut, W. GEAMANY

Libreien, Mex-Planck-Instiut, W. GERMANY

Prot. R.K. Janev, Inst of Physics, YUGOSLAVIA 

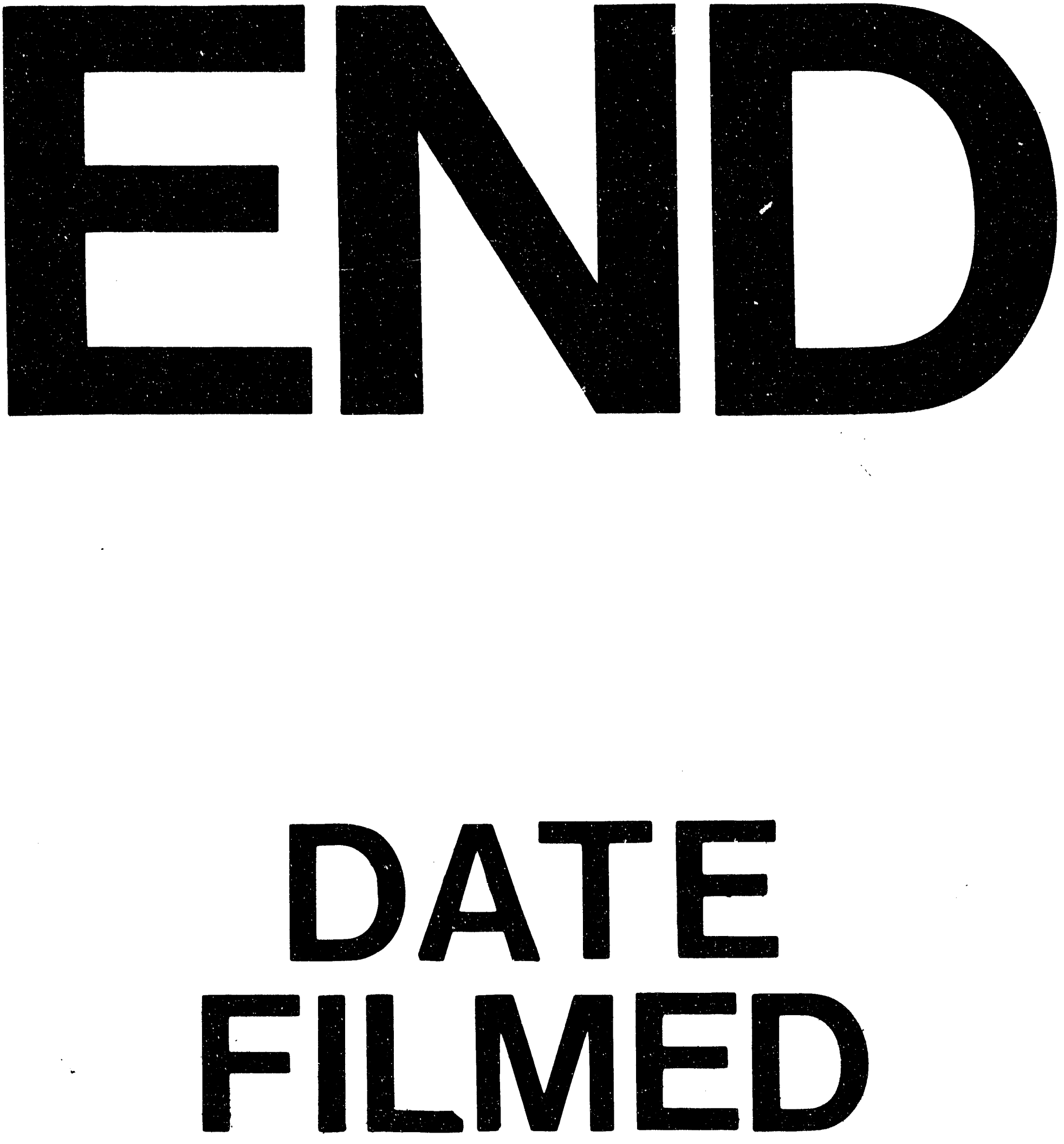

1

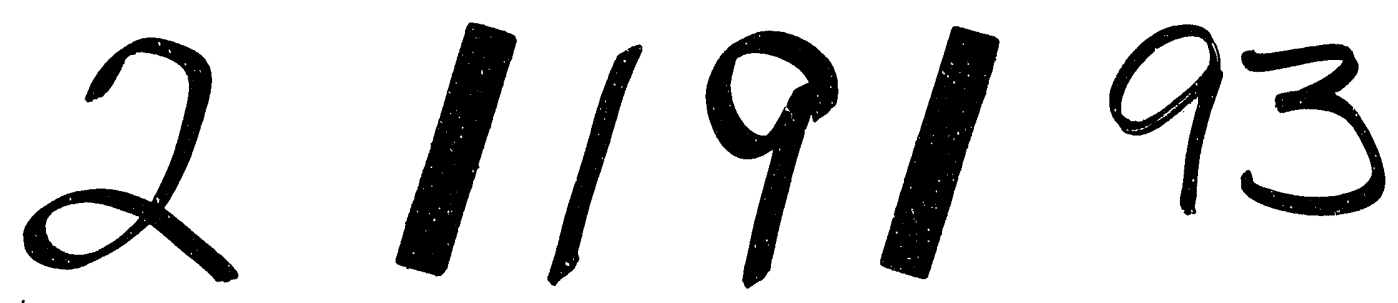


